\title{
Synthesis, characterization and thermal studies of transition metal complexes with O-, S-donor ligands
}

\begin{abstract}
Transition metal carboxylates have been synthesized with transition metal chlorides of $\mathrm{Cr}(\mathrm{III}), \mathrm{Mn}(\mathrm{II}), \mathrm{Fe}(\mathrm{III}), \mathrm{Co}(\mathrm{II}), \mathrm{Ni}(\mathrm{II}), \mathrm{Cu}(\mathrm{II})$ and $\mathrm{Ru}(\mathrm{III})$. The binding ligands are 3-[(4-bromophenylamido)]propenoic acid (L) and diethyl dithiocarbamate. All these compounds have been characterized by elemental analyses, conductivity measurements, UV/Vis, FT-IR spectroscopy and thermal analysis. Semi empirical studies of three compounds have also been carried out for structural elucidation showing that the $\mathrm{Cr}$ and Co derivatives have a six coordinated octahedral geometry which is in accordance with the data obtained from elemental analysis, UV/visible and FTIR spectroscopy. Thermal studies of all synthesized derivatives were carried out in a temperature range of 50$1000^{\circ} \mathrm{C}$ under inert atmosphere. Their kinetic parameters, like activation energy, enthalpy, entropy and order of reaction, were also studied. All complexes decompose at a certain temperature leaving behind metal oxides as residual products. The synthesized complexes showed remarkable anti-bacterial activities with a few exceptions. Selected compounds were also investigated for DNAdamage assay. The interaction of selected metal derivatives with DNA and their binding strength were investigated by UV/vis spectroscopy. The increasing order is $\left[\mathrm{CuL}\left(\mathrm{H}_{2} \mathrm{O}\right)_{2}\right] \mathrm{Cl}<\left[\mathrm{RuLL}^{\prime}\left(\mathrm{H}_{2} \mathrm{O}\right)_{2}\right] \mathrm{Cl}<\left[\mathrm{FeL}\left(\mathrm{H}_{2} \mathrm{O}\right)_{4}\right] \mathrm{Cl}_{2}<\left[\mathrm{CuLL}{ }^{\prime}<\right.$ 3 -[(4-bromophenylamido)] propenoic acid.
\end{abstract}

Keywords: metal complexes, 3-[(4-bromophenylamido)]propenoic acid, diethyl dithiocarbamate, IR, UV, TG, biological activities

\author{
Volume 5 Issue 6 - 2017 \\ Sadaf Ramzan, Samreen Saleem, Saqib Ali, \\ Saira Shahzadi, Saroj K Sharma, Kushal \\ Qunango, Bushra Mirza, Fiaz Ahmed \\ Department of Chemistry, Quaid-i-Azam University, \\ Islamabad-45320 Pakistan
}

Correspondence: Saqib Ali, Department of Chemistry, Quaidi-Azam University, Islamabad-45320 Pakistan,

Email sairashazadi@hotmail.com

Received: February 15, 2017 | Published: August 23, 2017
Abbreviations:OC, open circular; SC, supercoiled; DNA: deoxyribonucleic acid; $\mathrm{Cr}$, chromium; $\mathrm{Mn}$, manganese; Fe: iron; Co, cobalt; Ni: nickel; $\mathrm{Cu}$, copper; $\mathrm{Ru}$ : ruthenium; FTIR, fouriertransform infrared spectroscopy

\section{Introduction}

First row transition metal elements play important role in human life $\mathrm{e}^{1}$ and chemists are interested in co-ordination compounds for their application in industrial and synthetic processes, such as chemical, biological, environmental, ion-exchange catalysis, photochemistry, and biological applications, Moreover their thermal, spectral, microscopic and many other properties have also been examined. ${ }^{2-10}$

Metal carboxylates constitute an important class of compounds and receiving great attention due to their wide rang of application in the field of biomedicine and catalysis in the recent years. ${ }^{11}$ Literature shows that there are a wide variety of compounds of anions like dithiocarbamate, which have a corresponding mono thio analogue that is $\mathrm{S}, \mathrm{O}$ bound. ${ }^{12}$ Dithiocarbamates are usually made from sodium salts such as $\mathrm{NaS}_{2} \mathrm{CNMe}_{2}$ or by oxidations using thiuram disulphides, they can also be made by insertion reactions of $\mathrm{CS}_{2}$ with dialkyl amides. There is at present intense interest in the co-ordination of sulphur to metal ions, especially those of the $d$-transition series. ${ }^{13}$ Many of these complexes have unusual physical, chemical and structural properties. The $N, N$-diethyldithiocarbamate (dtc), has itself been widely used in the earlier transition metals ${ }^{14}$ in which, for example, the apparently simple complexes $M(\mathrm{dtc})_{2}$ ( $M$ (II) = chromium, manganese, iron, copper and zinc) actually involve 5-co-ordination. ${ }^{15}$ They has been found to act as a uninegative bidentate ligand, coordinating through both sulfur atoms, ${ }^{16}$ and both four- and six-coordinated complexes of a number of transition metal ions have been isolated. ${ }^{17}$ Dithiocarbamates and their complexes with transition metals have been used as common pesticides, vulcanizing or analytical agents for decades. ${ }^{12}$
In continuation of our interest in the synthesis seven transition metals complexes of HL have been prepared and characterized for the first time. Further we intend to investigate the spectroscopic behavior and kinetics of these complexes.

\section{Experimental}

\section{Materials and instrumentation}

The organic solvents (ethanol, acetone, chloroform) were used of Merck, Germany origin and dried in situ using standard procedures. ${ }^{18,19}$ All chemicals were of analytical grade and used without further purification. All transition metal chlorides were procured from Aldrich or Fluka. 3-[(4-Bromophenylamido)]propenoic acid was prepared in laboratory as reported earlier. ${ }^{20}$

Melting points were obtained in a capillary tube using an electrothermal melting point apparatus Mitamura Rikero Kogyo, Japan. FT-IR spectra of the compounds were recorded as $\mathrm{KBr}$ discs in the range of $4000-250 \mathrm{~cm}^{-1}$ on a Perkin-Elmer Spectrum; 1000 FT-IR Spectrophotometer. Atomic Absorption Spectra for transition metal estimations were recorded on an Analyst 300 of Perkin-Elmer. The conductance of the solutions of the complexes was determined from a 712 conductometer of Metrohm. Thermogravimetric analyses were carried out on a Perkin-Elmer, TGA-7 with computer interface. UV/ visible spectra were recorded on a Lambda $2 \mathrm{~S}$ of Perkin- Elmer.

\section{Procedure for the synthesis of transition metal complexes}

3-[(4-Bromophenylamido)]propenoic acid ( $1 \mathrm{mmol})$ was dissolved in anhydrous ethanol. Sodium bicarbonate $(1 \mathrm{mmol})$ was dissolved in deionized water and was added drop wise to the ethanolic solution of the ligand acid with constant stirring, until a clear solution was obtained. The solvent was evaporated on rotary evaporator and dried over calcium chloride (anhydrous). 


$$
\mathrm{R}-\mathrm{COOH}+\mathrm{NaHCO}_{3} \rightarrow \mathrm{R}-\mathrm{COONa}+\mathrm{CO}_{2}+\mathrm{H}_{2} \mathrm{O}
$$

Where $\mathrm{R}=$

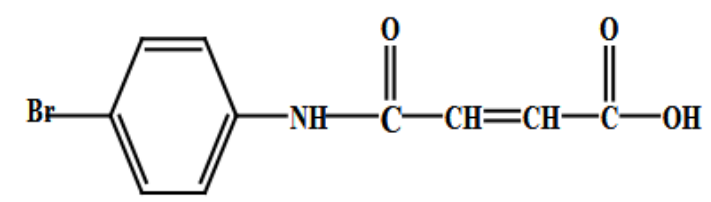

\section{3-[(4-Bromophenylamido)]propenoic acid.}

The sodium salt of the ligand acid $(1 \mathrm{mmol})$ was dissolved in anhydrous ethanol $(250 \mathrm{~mL})$ two-necked round bottom flask equipped with a water condenser and a magnetic stirrer bar. The transition metal chloride $(1 \mathrm{mmol})$ dissolved in ethanol was added drop by drop with constant stirring. The reaction mixture was refluxed for 3-4 hours in an inert atmosphere. The reaction mixture was allowed to stand overnight. Sodium chloride so formed was filtered off. The solvent was evaporated at room temperature. The solid mass left was recrystallized from a chloroform $/ n$ - hexane mixture (4:1). In the case of the Co complexes, $10 \mathrm{~mL}$ of $3 \% \mathrm{H}_{2} \mathrm{O}_{2}$ was also added in the reaction mixture.

The solid compound obtained was dissolved with an equimolar amount of the sodium salt of diethyldithiocarbamate (NaDTC) in ethanol. The reaction mixture was firstly stirred and then refluxed for three hours. The sodium salt formed was filtered off and the solvent was evaporated at room temperature.

$$
\begin{aligned}
& R-\mathrm{COONa}(\mathrm{EtOH})+\mathrm{MClx} \cdot n \mathrm{H}_{2} \mathrm{O}(\mathrm{EtOH}) \rightarrow \mathrm{M}(\mathrm{RCOO}) \mathrm{Clx}_{n} n \mathrm{H}_{2} \mathrm{O}+\mathrm{NaCl} \\
& \text { Reflux 3-4 hour. } \\
& M(\mathrm{RCOO}) \mathrm{Clx} \cdot n \mathrm{H}_{2} \mathrm{O}(\mathrm{EtOH})+\mathrm{NaDTC} \rightarrow(\mathrm{RCOO}) \mathrm{ClxDTC} \cdot n \mathrm{H}_{2} \mathrm{O}+\mathrm{NaCl} \\
& \text { Reflux } 3 \text { hour. } \\
& \text { Where } \\
& M=\mathrm{Cr}(I I I), \mathrm{Mn}(\mathrm{II}), \mathrm{Fe}(I I I), \mathrm{Co}(I I I), \mathrm{Ni}(I I), \mathrm{Cu}(\mathrm{II}) \text { and } \mathrm{Ru}(I I I) \\
& x=2,3 \text { and } n=2,4,6 . \\
& \text { DTC }=\text { Diethyldithiocarbamate }
\end{aligned}
$$

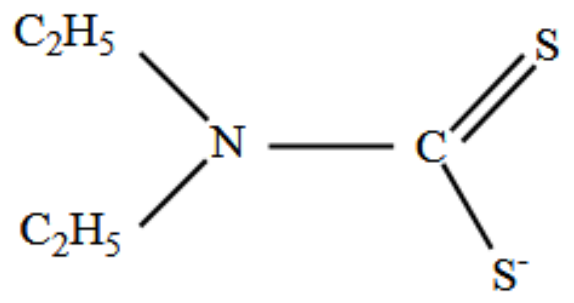

\section{Results and discussion}

3-[(4-Bromophenylamido)] propenoic acid is a brown odorless crystalline solid soluble in ethanol, methanol and acetone whereas insoluble in toluene and chloroform. It has the general formula $\mathrm{C}_{10} \mathrm{H}_{8} \mathrm{NO}_{3} \mathrm{Br}$ and a melting point of $270^{\circ} \mathrm{C}$. All newly synthesized compounds are semisolids. They are stable in air and are soluble in common organic solvents. The compounds have been characterized by IR and UV/Vis. spectroscopy to ascertain their structure and explore their properties. Selected complexes have also been screened for their biological activities and their interactions with DNA were also studied. The physical data of the compounds are reported in the experimental part along with the syntheses of the individual compounds. The physical data of the synthesized complexes are given in Table 1. All the compounds show high melting points. Elemental $(\mathrm{C}, \mathrm{H}$ and $\mathrm{N}$ ) and metal analysis data show good agreement with the calculated and found values (Table 2).

\section{UV/visible studies}

The UV/Vis absorptions of the complexes have been examined in the region of $800-200 \mathrm{~nm}$ with the object of ascertaining whether the spectra are compatible with those reported for the free ligand. The electronic spectra for the ligand and its metal derivatives were recorded in absolute ethanol and the results are described in Table 3. The sodium salt of 3-[(4-bromophenylamido)] propenoic acid does not show bands in the $400-800 \mathrm{~nm}$ regions. The UV/Vis. spectra of the $\mathrm{Cr}$ and $\mathrm{Co}$ complexes exhibit octahedral geometry. The spectra of the manganese complexes suggest a tetrahedral structure whereas Ni complex exhibit a square planar geometry. The spectra of the Fe complexes show two absorption peaks that can be attributed to a distorted octahedral geometry. Copper complexes show one band which corresponds to the square planar stereochemistry of the complex. The ruthenium complexes show only the charge transfer bands of an octahedral geometry.

In almost all complexes, some bands are derived from inter ligand and charge transfer or $\mathrm{n} \rightarrow \pi *$ transitions with decreasing energies and intensities. The bands near $35087 \mathrm{~cm}^{-1}(285 \mathrm{~nm})$ and $37037 \mathrm{~cm}^{-}$ ${ }^{1}(270 \mathrm{~nm})$ are assigned to inter ligand $\pi \rightarrow \pi *$ transitions for the aromatic moiety of the ligand. Moreover, a few sharp absorption bands are observed in the region $41666 \mathrm{~cm}^{-1}(240 \mathrm{~nm})-37037 \mathrm{~cm}^{-1}$ $(270 \mathrm{~nm})$ in the spectra of the complexes which could be assigned to charge transfer $\mathrm{L} \rightarrow \mathrm{M}$ or $\mathrm{n} \rightarrow \pi$ * transitions.

\section{Infrared spectroscopy}

Infrared spectra of the investigated compounds, the ligand acid and the salt of the ligand acid have been recorded as $\mathrm{KBr}$ pellets in the range of $4000-250 \mathrm{~cm}^{-1}$. The IR spectra of the complexes were compared with the spectrum of free ligands to determine the changes that might have taken place during complexation. The important bands and assignments of ligands and their complexes are summarized in Table 4 . In the $3500-2900 \mathrm{~cm}^{-1}$ region, ligand exhibit moderately intense bands typical of $\mathrm{OH}$ stretching vibrations, which were absent from the spectra of the complexes, indicating the coordination of a deprotonated $\mathrm{COO}$ - group to the central metal atom. The $\mathrm{C}=\mathrm{O}$ stretching vibrations of $\mathrm{HL}$ were observed at $1708 \mathrm{~cm}^{-1}$. The bands are intense and sharp. The difference between $v \mathrm{COO}_{\text {assm }}$ and $v \mathrm{COO}_{s v m}$ is important in the prediction of the nature of the binding mode of the ligand. ${ }^{21-23}$ The difference between the two vibration frequencies was in the range of $142-198 \mathrm{~cm}^{-1}$, suggesting a bidentate coordination mode of the COO- in the complexes. ${ }^{24}$ In all complexes, moderate to weak bands in the region of $425-495 \mathrm{~cm}^{-1}$ appear which are characteristic of $\mathrm{M}-\mathrm{O}$ bond.

In the case of the diethyldithiocarbamate complexes with transition metals of 3-[(4-bromophenylamido)] propenoic acid, additional bands appear in the FT-IR spectra. A characteristic feature of the dialkyldithiocarbamate group is the $\mathrm{C}-\mathrm{N}$ single bond stretching frequencies in amines in the region of $1250-1350 \mathrm{~cm}^{-1}$, close to the $\mathrm{C}-\mathrm{H}$ deformation bands. A single sharp band of high intensity of C-S has been observed around $1000 \mathrm{~cm}^{-1}$, indicating the bidentate binding of the dithiocarbamate. ${ }^{25} \mathrm{~A}$ band of medium intensity is characteristic for $v \mathrm{M}-\mathrm{S}$ in the $505-445 \mathrm{~cm}^{-1}$ range indicating that the $\mathrm{S}$ atom has been coordinated to the metal atom. ${ }^{26}$ 


\section{Thermogravimetric assay}

The thermal analysis of transition metal complexes was carried out by thermogravimetric technique. This technique also provides information about the kinetic parameters, such as order of reaction $(n)$, activation energy $\left(E^{*}\right)$, enthalpy $\left(\Delta H^{*}\right)$ and entropy $\left(\Delta S^{*}\right)$ through the rate of decomposition of the reactants with the temperature as a function of time $\left(20^{\circ} \mathrm{Cmin}^{-1}\right)$. The compounds were heated from ambient temperature to $1000 \mathrm{OC}$ under inert atmosphere. The interpretation of the thermogram was done with the Horowitz and Metzger method $[27,28]$ to find various kinetic parameters.
The ligand acid, 3-[(4-bromophenylamido)]propenoic acid (HL) decomposes in the temperature range of $190-310^{\circ} \mathrm{C}$ leaving traces of ash as residue with the order 0.92 and an activation energy of $10.43 \mathrm{KJ} /$ mole. The enthalpy of the reaction is $8.48 \mathrm{KJ} / \mathrm{mol}$ and the entropy is $-198.03 \mathrm{~J} / \mathrm{molK}$. Most compounds show a single step decomposition behavior while in the case of mixed ligand, the decomposition pattern encompasses on two to three decomposition steps with different kinetic parameters. The results of the kinetic parameters are mentioned in Table 5 .

Table I Physical data of Transition metal complexes of 3-[(4-Bromophenylamido)] propenoic acid and Diethyldithiocarbamate

\begin{tabular}{lllll}
\hline Compound & Mol. Weight & Color & Yield (\%) & Conductance $\boldsymbol{\mu s / c m}$ \\
\hline $\mathrm{HL}$ & 270 & Dark brown & -- & 0.08 \\
$\left.\left[\mathrm{CrL}\left(\mathrm{H}_{2} \mathrm{O}\right)_{3} \mathrm{Cl}\right)\right] \mathrm{Cl}$ & 446.0 & Green & 93 & 53.3 \\
{$\left[\mathrm{CrLL}\left(\mathrm{H}_{2} \mathrm{O}\right) \mathrm{Cl}\right]$} & 522.8 & Green & 71 & 32.6 \\
{$\left[\mathrm{MnL}\left(\mathrm{H}_{2} \mathrm{O}\right)_{2}\right] \mathrm{Cl}$} & 395.5 & Light yellow & 96 & 64.1 \\
{$[\mathrm{MnLL}]$} & 472.2 & Brownish yellow & 82 & 33.40 \\
{$\left[\mathrm{FeL}\left(\mathrm{H}_{2} \mathrm{O}\right)_{4}\right] \mathrm{Cl}_{2}$} & 467.9 & Yellow & 93 & 92.3 \\
{$\left[\mathrm{FeLL}\left(\mathrm{H}_{2} \mathrm{O}\right)_{2}\right] \mathrm{Cl}$} & 544.7 & Dark yellow & 80 & 42.90 \\
{$\left[\mathrm{CoL}\left(\mathrm{H}_{2} \mathrm{O}\right)_{2} \mathrm{Cl}\right]$} & 434.9 & Purple & 87 & 34.6 \\
{$\left[\mathrm{CoLL}\left(\mathrm{H}_{2} \mathrm{O}\right) \mathrm{Cl}\right]$} & 529.7 & Dark purple & 77 & 24.50 \\
{$\left[\mathrm{NiL}\left(\mathrm{H}_{2} \mathrm{O}\right)_{2}\right] \mathrm{Cl}$} & 399.2 & Green & 92 & 56.9 \\
{$[\mathrm{NiLL} ']$} & 476.0 & Blackish green & 73 & 33.84 \\
{$\left[\mathrm{CuL}\left(\mathrm{H}_{2} \mathrm{O}\right)_{2}\right] \mathrm{Cl}$} & 404.1 & Parrot green & 92 & 52.3 \\
{$[\mathrm{CuLL} ']$} & 480.8 & Blackish green & 76 & 36.70 \\
{$\left[\mathrm{RuL}\left(\mathrm{H}_{2} \mathrm{O}\right)_{4}\right] \mathrm{Cl}$} & 513.1 & Black & 79 & 61.5 \\
{$\left[\mathrm{RuLL}\left(\mathrm{H}_{2} \mathrm{O}\right)_{2}\right] \mathrm{Cl}$} & 589.9 & Black & 68 & 45.5
\end{tabular}

L:3-[(4-Bromophenylamido)]propenoic acid.

L': Diethyldithiocarbamate.

Table 2 Concentration of Metal, Carbon, Hydrogen and Nitrogen in Transition metal complexes of 3-[(4-Bromophenylamido)] propenoic acid and Diethyldithiocarbamate

\begin{tabular}{lllll}
\hline Compound & $\begin{array}{l}\text { Metal (\%) } \\
\text { Actual/Calc. }\end{array}$ & $\begin{array}{l}\text { Carbon (\%) } \\
\text { Actual/Calc. }\end{array}$ & $\begin{array}{l}\text { Hydrogen (\%) } \\
\text { Actual/Calc. }\end{array}$ & Nitrogen (\%) Actual/Calc. \\
\hline$\left.\left[\mathrm{CrL}\left(\mathrm{H}_{2} \mathrm{O}\right)_{3} \mathrm{Cl}\right)\right] \mathrm{Cl}$ & $11.23 / 11.66$ & $26.48 / 26.93$ & $2.82 / 2.94$ & $3.11 / 3.14$ \\
{$\left[\mathrm{CrLL}\left(\mathrm{H}_{2} \mathrm{O}\right) \mathrm{Cl}\right]$} & $10.01 / 9.95$ & $33.38 / 34.46$ & $3.82 / 3.66$ & $4.91 / 5.36$ \\
{$\left[\mathrm{MnL}\left(\mathrm{H}_{2} \mathrm{O}\right)_{2}\right] \mathrm{Cl}$} & $15.01 / 13.89$ & $31.48 / 30.37$ & $2.12 / 2.80$ & $3.82 / 3.54$ \\
{$[\mathrm{MnLL}]$} & $11.09 / 11.63$ & $37.64 / 38.15$ & $4.04 / 3.63$ & $5.89 / 5.93$ \\
{$\left[\mathrm{FeL}\left(\mathrm{H}_{2} \mathrm{O}\right)_{4}\right] \mathrm{Cl}{ }_{2}$} & $11.52 / 11.94$ & $25.17 / 25.67$ & $2.91 / 3.23$ & $2.68 / 2.99$ \\
{$\left[\mathrm{FeLL}\left(\mathrm{H}_{2} \mathrm{O}\right)_{2}\right] \mathrm{Cl}$} & $10.34 / 10.25$ & $33.06 / 33.08$ & $3.63 / 3.89$ & $5.29 / 5.14$ \\
{$\left[\mathrm{CoL}\left(\mathrm{H}_{2} \mathrm{O}\right)_{2} \mathrm{Cl}\right]$} & $13.16 / 13.55$ & $27.17 / 27.61$ & $2.12 / 2.55$ & $3.33 / 3.22$ \\
{$\left[\mathrm{CoLL}\left(\mathrm{H}_{2} \mathrm{O}\right) \mathrm{Cl}\right]$} & $10.96 / / 11.12$ & $33.45 / 34.01$ & $3.19 / 3.62$ & $5.22 / 5.29$ \\
{$\left[\mathrm{NiL}\left(\mathrm{H}_{2} \mathrm{O}\right)_{2}\right] \mathrm{Cl}$} & $14.39 / 14.70$ & $29.52 / 30.08$ & $2.27 / 2.78$ & $3.53 / 3.51$ \\
{$\left[\mathrm{NiLL}^{\prime}\right]$} & $11.87 / 12.33$ & $37.87 / 37.85$ & $3.56 / 3.60$ & $6.11 / 5.88$ \\
{$\left[\mathrm{CuL}\left(\mathrm{H}_{2} \mathrm{O}\right)_{2}\right] \mathrm{Cl}$} & $16.11 / 15.73$ & $29.9 / 29.72$ & $2.58 / 2.74$ & $3.53 / 3.47$ \\
{$[\mathrm{CuLL}]$} & $13.91 / 13.21$ & $38.02 / 37.46$ & $3.65 / 3.56$ & $5.70 / 5.83$ \\
{$\left[\mathrm{RuL}\left(\mathrm{H}_{2} \mathrm{O}\right)_{4}\right] \mathrm{Cl}$} & $20.22 / 19.74$ & $25.44 / 23.41$ & $2.69 / 2.95$ & $3.06 / 2 . .73$ \\
{$\left[\mathrm{RuLL}^{\prime}\left(\mathrm{H}_{2} \mathrm{O}\right)_{2}\right] \mathrm{Cl}$} & $17.44 / 17.14$ & $29.95 / 30.54$ & $3.02 / 3.59$ & $4.88 / 4.75$ \\
\hline
\end{tabular}

$L: 3-[(4-B r o m o p h e n y l a m i d o)]$ propenoic acid

L': Diethyldithiocarbamate.

Table 3 Absorption Pattern of Transition Metal Complexes of 3-[(4-Bromophenylamido)] propenoic acid and Diethyldithiocarbamate

\begin{tabular}{llll}
\hline Compound & $\lambda \mathbf{c m}^{-1}(\mathbf{n m})$ & Electronic Transition & Geometry \\
\hline $\left.\left.\mathrm{CrL}\left(\mathrm{H}_{2} \mathrm{O}\right){ }_{3} \mathrm{Cl}\right)\right] \mathrm{Cl}$ & $16452(608)$ & ${ }^{4} \mathrm{~A}_{2} \mathrm{~g}(\mathrm{~F}) \rightarrow{ }^{4} \mathrm{~T}_{2} \mathrm{~g}(\mathrm{~F})$ & \\
& $26984(37 \mathrm{I})$ & ${ }^{4} \mathrm{~A}_{2} \mathrm{~g}(\mathrm{~F}) \rightarrow{ }^{4} \mathrm{~T}_{1} \mathrm{~g}(\mathrm{~F})$ & Octahedral \\
& $34503(290)$ & ${ }^{4} \mathrm{~A}_{2} \mathrm{~g}(\mathrm{~F}) \rightarrow{ }^{4} \mathrm{~T}_{1} \mathrm{~g}(\mathrm{P})$ & \\
{$\left[\mathrm{CrLL}^{\prime}\left(\mathrm{H}_{2} \mathrm{O}\right) \mathrm{Cl}\right]$} & $1592 \mathrm{I}(628)$ & ${ }^{4} \mathrm{~A}_{2} \mathrm{~g}(\mathrm{~F}) \rightarrow{ }^{4} \mathrm{~T}_{2} \mathrm{~g}(\mathrm{~F})$ & \\
& $27818(360)$ & $\left.{ }^{4} \mathrm{~A}_{2} \mathrm{~g} \mathrm{~F}\right) \rightarrow{ }^{4} \mathrm{~T}_{1} \mathrm{~g}(\mathrm{~F})$ & Octahedral
\end{tabular}


Table Continued...

\begin{tabular}{|c|c|c|c|}
\hline Compound & $\lambda \mathrm{cm}^{-1}(\mathrm{~nm})$ & Electronic Transition & Geometry \\
\hline$\left[\mathrm{MnL}\left(\mathrm{H}_{2} \mathrm{O}\right)_{2}\right] \mathrm{Cl}$ & $\begin{array}{l}16129(620) \\
23310(429) \\
31645(316)\end{array}$ & $\begin{array}{l}{ }^{6} \mathrm{~A}_{1} \mathrm{~g} \rightarrow{ }^{4} \mathrm{~T}_{1} \mathrm{~g} \\
{ }^{6} \mathrm{~A}_{1} \mathrm{~g} \rightarrow{ }^{4} \mathrm{Eg}{ }^{4} \mathrm{~T}_{1} \mathrm{~g} \\
{ }^{6} \mathrm{~A}_{1} \mathrm{~g} \rightarrow{ }^{4} \mathrm{Eg}\end{array}$ & Tetrahedral \\
\hline [MnLL'] & $\begin{array}{l}15879(630) \\
24839(403) \\
31435(318)\end{array}$ & $\begin{array}{l}{ }^{6} \mathrm{~A}_{1} \mathrm{~g} \rightarrow{ }^{4} \mathrm{~T}_{1} \mathrm{~g} \\
{ }^{6} \mathrm{~A}_{1} \mathrm{~g} \rightarrow{ }^{4} \mathrm{Eg}{ }^{4} \mathrm{~T}_{1} \mathrm{~g} \\
{ }^{6} \mathrm{~A}_{1} \mathrm{~g} \rightarrow{ }^{4} \mathrm{Eg}\end{array}$ & Tetrahedral \\
\hline$\left[\mathrm{FeL}\left(\mathrm{H}_{2} \mathrm{O}\right)_{4}\right] \mathrm{Cl}_{2}$ & $\begin{array}{l}20325(492) \\
24964(401)\end{array}$ & $\begin{array}{l}{ }^{6} \mathrm{~A}_{1} \mathrm{~g} \rightarrow{ }^{4} \mathrm{~T}_{1} \mathrm{~g}(\mathrm{G}) \\
{ }^{6} \mathrm{~A}_{1} \mathrm{~g} \rightarrow{ }^{4} \mathrm{~T}_{2} \mathrm{~g}(\mathrm{G})\end{array}$ & Octahedral \\
\hline$\left[\mathrm{FeLL}^{\prime}\left(\mathrm{H}_{2} \mathrm{O}\right)_{2}\right] \mathrm{Cl}$ & $\begin{array}{l}19527(512) \\
25325(395)\end{array}$ & $\begin{array}{l}{ }^{6} \mathrm{~A}_{1} \mathrm{~g} \rightarrow{ }^{4} \mathrm{~T}_{1} \mathrm{~g}(\mathrm{G}) \\
{ }^{6} \mathrm{~A}_{1} \mathrm{~g} \rightarrow{ }^{4} \mathrm{~T}_{2} \mathrm{~g}(\mathrm{G})\end{array}$ & Octahedral \\
\hline$\left[\mathrm{CoL}\left(\mathrm{H}_{2} \mathrm{O}\right)_{2} \mathrm{Cl}_{2}\right]$ & $\begin{array}{l}|605|(623) \\
23529(425)\end{array}$ & $\begin{array}{l}{ }^{\mathrm{A}} \mathrm{A}_{1} \mathrm{~g} \rightarrow{ }^{\prime} \mathrm{T}_{1} \mathrm{~g} \\
{ }^{\mathrm{I}} \mathrm{A}_{1} \mathrm{~g} \rightarrow{ }^{\prime} \mathrm{T}_{2} \mathrm{~g}\end{array}$ & Octahedral \\
\hline$\left[\operatorname{CoLL}^{\prime}\left(\mathrm{H}_{2} \mathrm{O}\right) \mathrm{Cl}\right]$ & $\begin{array}{l}16260(615) \\
23041(434)\end{array}$ & $\begin{array}{l}{ }^{ } \mathrm{A}_{1} \mathrm{~g} \rightarrow{ }^{\prime} \mathrm{T}_{1} \mathrm{~g} \\
{ }^{\mathrm{I}} \mathrm{A}_{1} \mathrm{~g} \rightarrow{ }^{\prime} \mathrm{T}_{2} \mathrm{~g}\end{array}$ & Octahedral \\
\hline$\left[\mathrm{NiL}\left(\mathrm{H}_{2} \mathrm{O}\right)_{2}\right] \mathrm{Cl}$ & $\begin{array}{l}18734(534) \\
23809(420)\end{array}$ & $\begin{array}{l}{ }^{3} A^{2} g \rightarrow{ }^{3} T_{1} g(F) \\
{ }^{3} A^{2} g \rightarrow{ }^{3} T_{1} g(P)\end{array}$ & Square planner \\
\hline [NiLL'] & $\begin{array}{l}19834(504) \\
25189(397)\end{array}$ & $\begin{array}{l}{ }^{3} A^{2} g \rightarrow{ }^{3} T_{1} g(F) \\
{ }^{3} A^{2} g \rightarrow{ }^{3} T_{1} g(P)\end{array}$ & Square planner \\
\hline$\left[\mathrm{CuL}\left(\mathrm{H}_{2} \mathrm{O}\right)_{2}\right] \mathrm{Cl}$ & $25437(393)$ & ${ }^{2} \mathrm{Eg} \rightarrow{ }^{2} \mathrm{~B}_{\mathrm{I}} \mathrm{g}$ & Square planner \\
\hline [CuLL'] & $24890(402)$ & ${ }^{2} \mathrm{Eg} \rightarrow{ }^{2} \mathrm{~B}_{\mathrm{I}} \mathrm{g}$ & Square planner \\
\hline$\left[\operatorname{RuL}\left(\mathrm{H}_{2} \mathrm{O}\right)_{4}\right] \mathrm{Cl}_{2}$ & $43405(230)$ & CT & Octahedral \\
\hline$\left[\mathrm{RuLL}^{\prime}\left(\mathrm{H}_{2} \mathrm{O}\right)_{2}\right] \mathrm{Cl}$ & $43925(228)$ & CT & Octahedral \\
\hline
\end{tabular}

L: 3-[(4-Bromophenylamido)]propenoic acid.

L': Diethyldithiocarbamate.

Table 4 IR spectral data (cm - I) for the Transition Metal Complexes of 3-[(4-Bromophenylamido)]propenoic acid and Diethyldithiocarbamate

\begin{tabular}{|c|c|c|c|c|c|c|c|c|c|}
\hline \multirow{2}{*}{ Compound } & \multirow{2}{*}{ v $\mathrm{C}=\mathrm{O}$} & \multicolumn{2}{|c|}{ vCOO } & \multirow{2}{*}{$-\Delta v$} & \multirow{2}{*}{$v \mathbf{N}-\mathbf{H}$} & \multirow{2}{*}{$v C=S$} & \multirow{2}{*}{$v \mathbf{C}-\mathbf{N}$} & \multirow{2}{*}{ v M-O } & \multirow{2}{*}{ v M-S } \\
\hline & & asym & sym & & & & & & \\
\hline HL & 1708 & $|54|$ & 1389 & 152 & 3266 & -- & -- & -- & -- \\
\hline $\mathrm{NaL}^{\prime}$ & 1710 & 1587 & |39| & 196 & 3258 & -- & -- & & -- \\
\hline$\left.\left[\mathrm{CrL}\left(\mathrm{H}_{2} \mathrm{O}\right)_{3} \mathrm{Cl}\right)\right] \mathrm{Cl}$ & 1709 & 1597 & 1399 & 198 & 3257 & -- & -- & 482 & -- \\
\hline$\left[\mathrm{CrLL}\left(\mathrm{H}_{2} \mathrm{O}\right) \mathrm{Cl}\right]$ & $|7| 1$ & 1579 & 1394 & 185 & 3284 & 1007 & 1247 & 432 & 500 \\
\hline$\left[\mathrm{MnL}\left(\mathrm{H}_{2} \mathrm{O}\right)_{2}\right] \mathrm{Cl}$ & 1739 & 1537 & 1353 & 184 & 3413 & -- & -- & 427 & -- \\
\hline [MnLL'] & 1745 & 1580 & 1397 & 183 & 3264 & 1002 & 1270 & 428 & 505 \\
\hline$\left[\mathrm{FeL}\left(\mathrm{H}_{2} \mathrm{O}\right)_{4}\right] \mathrm{Cl}_{2}$ & 1710 & 1535 & 1393 & 143 & 3359 & -- & -- & 438 & -- \\
\hline$\left[\right.$ FeLL' $\left.\left(\mathrm{H}_{2} \mathrm{O}\right)_{2}\right] \mathrm{Cl}$ & 1716 & 1534 & 1392 & 142 & 3254 & 1012 & 1264 & 481 & 508 \\
\hline$\left[\mathrm{CoL}\left(\mathrm{H}_{2} \mathrm{O}\right)_{2} \mathrm{Cl}_{2}\right]$ & 1735 & 1544 & 1358 & 186 & 3257 & -- & -- & 448 & -- \\
\hline [CoLL'( $\left.\left.\mathrm{H}_{2} \mathrm{O}\right) \mathrm{Cl}\right]$ & 1712 & 1540 & 1395 & 145 & 3278 & 1008 & 1250 & 432 & 465 \\
\hline$\left[\mathrm{NiL}\left(\mathrm{H}_{2} \mathrm{O}\right)_{2}\right] \mathrm{Cl}$ & $|74|$ & 1536 & 1355 & 181 & 3415 & -- & -- & 430 & -- \\
\hline [NiLL'] & 1745 & 1542 & 1396 & 146 & 3324 & 1008 & 1247 & 431 & 445 \\
\hline$\left[\mathrm{CuL}\left(\mathrm{H}_{2} \mathrm{O}\right)_{2}\right] \mathrm{Cl}$ & 1725 & 1536 & 1353 & 183 & 3413 & -- & -- & 425 & -- \\
\hline [CuLL'] & I7II & 1542 & 1392 & 150 & 3275 & 1009 & $125 \mid$ & 433 & 462 \\
\hline$\left[\mathrm{RuL}\left(\mathrm{H}_{2} \mathrm{O}\right)_{4}\right] \mathrm{Cl}_{2}$ & 1710 & 1536 & 1392 & 144 & 3254 & -- & -- & 495 & -- \\
\hline$\left[\mathrm{RuLL}^{\prime}\left(\mathrm{H}_{2} \mathrm{O}\right)_{2}\right] \mathrm{Cl}$ & 1716 & 1544 & 1390 & 154 & 3250 & 1009 & 1249 & 450 & 489 \\
\hline
\end{tabular}

L: 3-[(4-Bromophenylamido)] propenoic acid NaL': Sodium salt of diethyldithiocarbamate.

Table 5 Thermal Decomposition Pattern and Kinetic Parameters of Transition Metal Complexes of 3-[(4-Bromophenylamido)]propenoic acid and Diethyldithiocarbamate

\begin{tabular}{|c|c|c|c|c|c|c|c|}
\hline \multirow{2}{*}{ Compound } & \multirow{2}{*}{ Temp. ${ }^{\circ} \mathrm{C}$} & \multirow{2}{*}{ Order (n) } & \multirow{2}{*}{$\mathbf{E}^{*} \mathrm{~kJ} / \mathrm{mol}$} & \multirow{2}{*}{$\Delta \mathbf{H}^{*} \mathrm{~kJ} / \mathrm{mol}$} & \multirow{2}{*}{$\Delta \mathbf{S}^{*} \mathrm{~J} / \mathrm{molK}$} & \multicolumn{2}{|c|}{$\%$ wt. loss } \\
\hline & & & & & & cal. & obs. \\
\hline HL & $190-310$ & 0.92 & 10.435 & 8.48 & -198.03 & -- & 973 \\
\hline$\left[\mathrm{CrL}\left(\mathrm{H}_{2} \mathrm{O}{ }_{2} \mathrm{Cl}\right)\right] \mathrm{Cl}$ & $90-900$ & 1.13 & 10.15 & 7.70 & -226.12 & 81.2 & 83.5 \\
\hline$\left.\left[\mathrm{CrL}\left(\mathrm{I}_{2} \mathrm{C}\right)_{3} \mathrm{Cl}\right)\right]$ & $90-900$ & 1.13 & 10.10 & 1.10 & -226.12 & 18.8 & 16.5 \\
\hline \multirow[t]{2}{*}[\mathrm{CrLL}^{\prime}(\mathrm{H}_{2}\mathrm{O})\mathrm{Cl}]{} & $100-900$ & 1.06 & 8.816 & 4.99 & -272.37 & $\begin{array}{l}83.9 \\
16.1\end{array}$ & $\begin{array}{l}84.8 \\
15.2\end{array}$ \\
\hline & $100-440$ & 1.2 & 13.64 & 11.06 & -199.4 & 48.5 & 46.5 \\
\hline$\left[\mathrm{MnL}\left(\mathrm{H}_{2} \mathrm{O}\right)_{2}\right] \mathrm{Cl}$ & $700-900$ & 0.95 & 139.3 & 132.3 & 107.05 & $\begin{array}{l}29.5 \\
22.0\end{array}$ & $\begin{array}{l}29.7 \\
23.8\end{array}$ \\
\hline \multirow[t]{2}{*}{ [MnLL'] } & $100-900$ & 1.01 & 6.01 & 4.35 & -238.66 & $\begin{array}{l}81.6 \\
18.4\end{array}$ & $\begin{array}{l}80.2 \\
19.8\end{array}$ \\
\hline & $90-280$ & 1.06 & 12.91 & 11.09 & -159.93 & 39.2 & 40.0 \\
\hline$\left[\mathrm{FeL}\left(\mathrm{H}_{2} \mathrm{O}\right)_{4}\right] \mathrm{Cl}_{2}$ & $300-900$ & 1.06 & 26.54 & 20.63 & $\begin{array}{l}-137.75 \\
-217.65\end{array}$ & $\begin{array}{l}45.4 \\
15.4\end{array}$ & $\begin{array}{l}43.3 \\
16.7\end{array}$ \\
\hline
\end{tabular}


Table Continued..

\begin{tabular}{|c|c|c|c|c|c|c|c|}
\hline \multirow{2}{*}{ Compound } & \multirow{2}{*}{ Temp. ${ }^{\circ} \mathrm{C}$} & \multirow{2}{*}{ Order (n) } & \multirow{2}{*}{$\mathbf{E} * \mathrm{~kJ} / \mathrm{mol}$} & \multirow{2}{*}{$\Delta \mathbf{H}^{*} \mathrm{~kJ} / \mathrm{mol}$} & \multirow{2}{*}{$\Delta \mathbf{S}^{*} \mathrm{~J} / \mathrm{molK}$} & \multicolumn{2}{|c|}{$\%$ wt. loss } \\
\hline & & & & & & cal. & obs. \\
\hline$\left[F_{e L L}\left(\mathrm{H}_{2} \mathrm{O}\right)_{2}\right] \mathrm{Cl}$ & $100-900$ & 1.02 & 6.86 & 5.16 & -228.68 & $\begin{array}{l}86.8 \\
13.2\end{array}$ & $\begin{array}{l}85.5 \\
14.5\end{array}$ \\
\hline$\left[\mathrm{CoL}\left(\mathrm{H}_{2} \mathrm{O}\right)_{2} \mathrm{Cl}_{2}\right]$ & $110-660$ & I.I & 5.02 & 2.56 & -279.7 & $\begin{array}{l}80.4 \\
17.2\end{array}$ & $\begin{array}{l}81.7 \\
18.3\end{array}$ \\
\hline$\left[\mathrm{CoLL}^{\prime}\left(\mathrm{H}_{2} \mathrm{O}\right) \mathrm{Cl}\right]$ & $\begin{array}{l}90-400 \\
400-900\end{array}$ & $\begin{array}{l}1.18 \\
0.95\end{array}$ & $\begin{array}{l}8.29 \\
31.65\end{array}$ & $\begin{array}{l}6.26 \\
25.62\end{array}$ & $\begin{array}{l}-227.7 \\
-200.09\end{array}$ & $\begin{array}{l}45.4 \\
40.5 \\
14.1\end{array}$ & $\begin{array}{l}44.4 \\
39.2 \\
16.4\end{array}$ \\
\hline$\left[\mathrm{NiL}\left(\mathrm{H}_{2} \mathrm{O}\right)_{2}\right] \mathrm{Cl}$ & $90-700$ & I. 18 & 6.64 & 3.89 & -269.4 & $\begin{array}{l}81.3 \\
18.7\end{array}$ & $\begin{array}{l}80.4 \\
19.6\end{array}$ \\
\hline [NiLL'] & $60-550$ & 1.01 & 5.65 & 3.48 & -264 & $\begin{array}{l}84.3 \\
15.7\end{array}$ & $\begin{array}{l}83.2 \\
16.8\end{array}$ \\
\hline$\left[\mathrm{CuL}\left(\mathrm{H}_{2} \mathrm{O}\right)_{2}\right] \mathrm{Cl}$ & $200-900$ & 1.58 & 48.13 & 45.93 & $\mid 44.35$ & $\begin{array}{l}80.3 \\
19.7\end{array}$ & $\begin{array}{l}78.9 \\
21.1\end{array}$ \\
\hline [CuLL'] & $\begin{array}{l}100-500 \\
700-900\end{array}$ & $\begin{array}{l}1.07 \\
0.92\end{array}$ & $\begin{array}{l}6.17 \\
126.47\end{array}$ & $\begin{array}{l}4.09 \\
119.49\end{array}$ & $\begin{array}{l}-254.86 \\
69.82\end{array}$ & $\begin{array}{l}58.5 \\
25.0 \\
16.5\end{array}$ & $\begin{array}{l}57.9 \\
24.8 \\
17.3\end{array}$ \\
\hline$\left[\mathrm{RuL}\left(\mathrm{H}_{2} \mathrm{O}\right)_{4}\right] \mathrm{Cl}_{2}$ & $100-900$ & I.0I & 7.47 & 3.70 & -281 & $\begin{array}{l}73.4 \\
22.8\end{array}$ & $\begin{array}{l}76.1 \\
23.9\end{array}$ \\
\hline$\left[\operatorname{RuLL}^{\prime}\left(\mathrm{H}_{2} \mathrm{O}\right)_{2}\right] \mathrm{Cl}$ & $90-340$ & 1.22 & 10.80 & 8.88 & $-|91.4|$ & $\begin{array}{l}80.1 \\
19.9\end{array}$ & $\begin{array}{l}78.6 \\
21.4\end{array}$ \\
\hline
\end{tabular}

L: 3-[(4-Bromophenylamido)]propenoic acid.

L': Diethyldithiocarbamate.

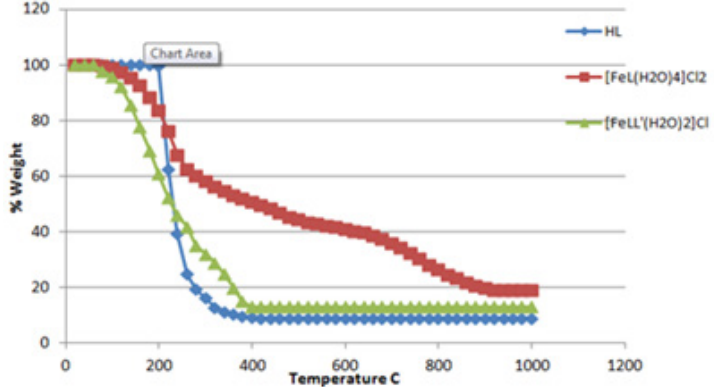

Figure I Thermal decomposition pattern of 3-[(4-bromophenylamido)] propenoic acid, $\left[\mathrm{FeL}\left(\mathrm{H}_{2} \mathrm{O}\right)_{4}\right] \mathrm{Cl}_{2}$ and $\left[\mathrm{FeLL}\left(\mathrm{H}_{2} \mathrm{O}\right)_{2}\right] \mathrm{Cl}$.

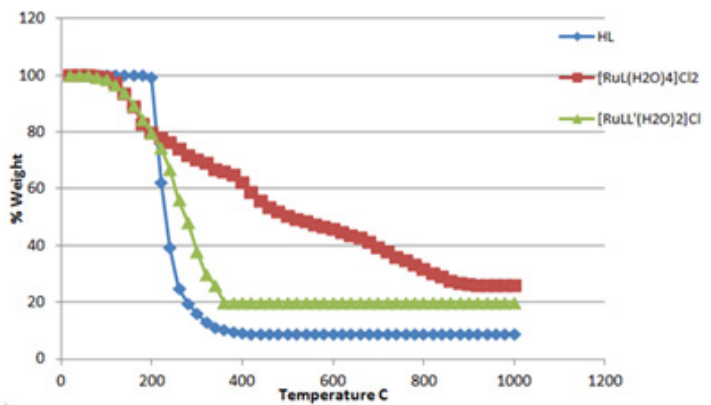

Figure 2 Thermal decomposition pattern of 3-[(4-bromophenylamido)] propenoic acid, $\left[\operatorname{RuL}\left(\mathrm{H}_{2} \mathrm{O}\right)_{4}\right] \mathrm{Cl}_{2}$ and $\left[\operatorname{RuLL}\left(\mathrm{H}_{2} \mathrm{O}\right)_{2}\right] \mathrm{Cl}$.

All synthesized complexes decompose at higher temperature and left behind the oxides of the respective metals. The kinetic values described in Table 5 reflect the stability of the complexes against the given temperature. Figure $1 \& 2$ show the thermal behavior of compounds $\left[\mathrm{FeL}\left(\mathrm{H}_{2} \mathrm{O}\right)_{4}\right] \mathrm{Cl}_{2},\left[\mathrm{FeLL}\left(\mathrm{H}_{2} \mathrm{O}\right)_{2}\right] \mathrm{Cl}$ and $\left[\mathrm{RuL}\left(\mathrm{H}_{2} \mathrm{O}\right)_{4}\right]$ $\mathrm{Cl}_{2},\left[\mathrm{RuLL}^{\prime}\left(\mathrm{H}_{2} \mathrm{O}\right)_{2}\right] \mathrm{Cl}$ respectively over the temperature range of 50 $1000^{\circ} \mathrm{C}$ under inert atmosphere, whereas Figure $3 \& 4$ show the plots of $\theta$ vs. $\ln \ln \mathrm{W}^{\circ} / \mathrm{W}$ for thermogravimatric analyses for decomposition of compounds $\left[\mathrm{RuL}\left(\mathrm{H}_{2} \mathrm{O}\right)_{4}\right] \mathrm{Cl}_{2},\left[\mathrm{RuLL}^{\prime}\left(\mathrm{H}_{2} \mathrm{O}\right)_{2}\right] \mathrm{Cl}$, respectively.

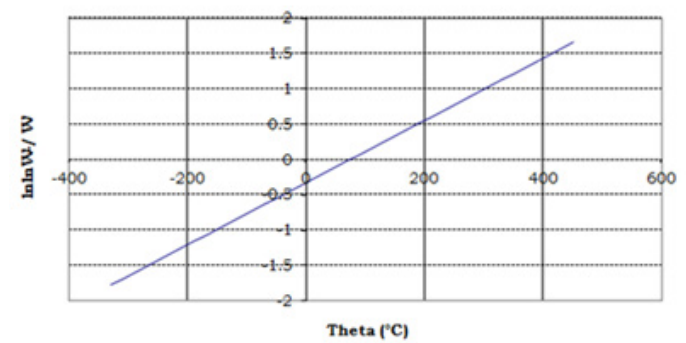

Figure 3 Plot of theta vs. InlnW $/ \mathrm{W}$ for thermogravimatric analysis for decomposition of $\left[\mathrm{RuL}\left(\mathrm{H}_{2} \mathrm{O}\right)_{4}\right] \mathrm{Cl}_{2}$.

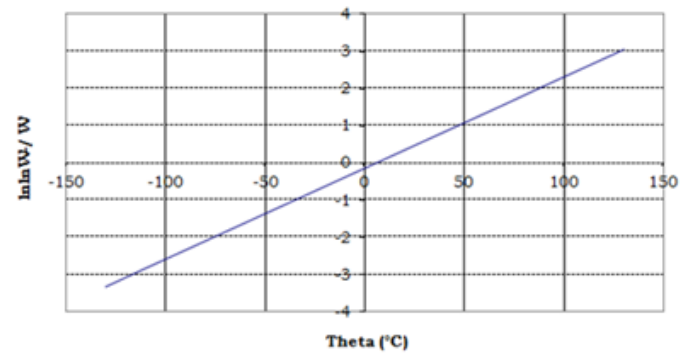

Figure 4 Plot of theta vs. Inln $W^{\circ} / \mathrm{W}$ for thermogravimatric analysis for decomposition of $\left[\mathrm{RuLL}^{\prime}\left(\mathrm{H}_{2} \mathrm{O}\right)_{2}\right] \mathrm{Cl}$.

\section{Biological activity}

Antibacterial assay: 3-[(4-Bromophenylamido)] propenoic acid is capable of inhibiting bacterial growth and activity by interfering with the metabolic process in the bacteria. In the present work, the antibacterial activities of these complexes have been determined against four strains of bacteria (Staphylococcus aureus (ATCC 25923), Bacillus subtilis (DSM 3256), Escherichia coli (ATCC 25922), and Pseudomonas aureginosa (ATCC 10197). Significant antibacterial activities were observed as compared to a standard drug. The metal complex $\left[\mathrm{FeL}\left(\mathrm{H}_{2} \mathrm{O}\right)_{4}\right] \mathrm{Cl}_{2}$ exhibited very good activity 
against two bacteria Staphylococcus aureus and Pseudomonas aureginosawhile it has relatively low activity against Bacillus subtilis and Escherichia coli. The metal complexes of $\left[\mathrm{RuL}\left(\mathrm{H}_{2} \mathrm{O}\right)_{4}\right]$ $\mathrm{Cl}_{2}$ exhibited good activity against Bacillus subtilis and Pseudomonas aureginosa, while it showed moderate activity against Staphylococcus aureus and Escherichia coli. Compound $\left[\mathrm{CuL}\left(\mathrm{H}_{2} \mathrm{O}\right)_{2}\right] \mathrm{Cl}$ showed good activity against only Staphylococcus aureus, while it showed very low activities against all other three bacteria. The metal complexes $[\mathrm{CuLL}]$ and $\left[\mathrm{RuLL}\left(\mathrm{H}_{2} \mathrm{O}\right)_{2}\right] \mathrm{Cl}$ showed moderate activity against all four bacteria, while $\left[\mathrm{FeLL}^{\prime}\left(\mathrm{H}_{2} \mathrm{O}\right)_{2}\right] \mathrm{Cl}$ are ineffective against all four bacteria. The results are described in Table 6 (Figure 5).

Table 6 Antibacterial Assay of Selected Transition Metal Complexes of 3-[(4-Bromophenylamido)]propenoic acid and Diethyldithiocarbamate

\begin{tabular}{lllll}
\hline \multirow{2}{*}{ Std./ Compound } & S. aureusATCC 25923 & B. subtilisDSM 3256 & E. coliATCC 25922 & P. aureginosaATCC I0I97 \\
\cline { 2 - 5 } & \% Inhibition & \% Inhibition & \% Inhibition & \% Inhibition \\
\hline Imipenum & $100(18 \mathrm{~mm})$ & $100(16 \mathrm{~mm})$ & $100(18 \mathrm{~mm})$ & $100(2 \mathrm{~mm})$ \\
$\mathrm{HL}$ & 24 & 11 & 31 & 22 \\
{$\left[\mathrm{FeL}\left(\mathrm{H}_{2} \mathrm{O}\right)_{4}\right] \mathrm{Cl}_{2}$} & 48 & 17 & 38 & 50 \\
{$\left[\mathrm{FeLL}\left(\mathrm{H}_{2} \mathrm{O}\right)_{2}\right] \mathrm{Cl}$} & 00 & 00 & 00 & 00 \\
{$\left[\mathrm{CuL}\left(\mathrm{H}_{2} \mathrm{O}\right)_{2}\right] \mathrm{Cl}$} & 48 & 11 & 19 & 28 \\
{$[\mathrm{CuLL}]$} & 24 & 28 & 25 & 33 \\
{$\left[\mathrm{RuL}\left(\mathrm{H}_{2} \mathrm{O}\right)_{4}\right] \mathrm{Cl}$} & 43 & 50 & 25 & 50 \\
{$\left[\mathrm{RuLL}^{\prime}\left(\mathrm{H}_{2} \mathrm{O}\right)_{2}\right] \mathrm{Cl}$} & 33 & 33 & 44 & 39
\end{tabular}

$\mathrm{HL}=\mathrm{L}: 3-[(4-$ Bromophenylamido $)]$ propenoic acid.

L': Diethyldithiocarbamate

Table 7 DNA Protection Activity of Transition Metal Complexes of 3-[(4-Bromophenylamido)]propenoic acid and Diethyldithiocarbamate

\begin{tabular}{llll}
\hline Compound & Concentration (ppm) & Protection & Damage \\
$\mathrm{HL}$ & 1000 & +++ & - \\
& 100 & +++ & - \\
{$\left[\mathrm{FeL}\left(\mathrm{H}_{2} \mathrm{O}\right)_{4}\right] \mathrm{Cl}_{2}$} & 10 & +++ & +++ \\
& 1000 & - & +++ \\
{$\left[\mathrm{FeLL}\left(\mathrm{H}_{2} \mathrm{O}\right)_{2}\right] \mathrm{Cl}$} & 100 & -+++ & - \\
& 10 & +++ & - \\
& 1000 & +++ & +++ \\
{$\left[\mathrm{CuL}\left(\mathrm{H}_{2} \mathrm{O}\right)_{2}\right] \mathrm{Cl}$} & 100 & +++ & - \\
& 10 & - & +++ \\
{$[\mathrm{CuLL}$} & 1000 & +++ & - \\
& 100 & +++ & - \\
& 10 & - & ++ \\
{$\left[\mathrm{RuL}\left(\mathrm{H}_{2} \mathrm{O}\right)_{4}\right] \mathrm{Cl}$} & 1000 & +++ & - \\
& 100 & +++ & - \\
{$\left[\mathrm{RuLL}\left(\mathrm{H}_{2} \mathrm{O}\right)_{2}\right] \mathrm{Cl}$} & 10 & - & - \\
& 1000 & +++ & - \\
& 100 & +++ & - \\
\hline
\end{tabular}

L: 3-[(4-Bromophenylamido)]propenoic acid.

L': Diethyldithiocarbamate.

+ :Weakly effective.

++ : Moderately effective.

+++ : Strongly effective.

Table 8 Interaction of Transition Metal Complexes of 3-[(4-Bromophenylamido)]propenoic acid and Diethyldithiocarbamate with Different Concentrations of DNA

\begin{tabular}{|c|c|c|c|c|c|c|c|c|c|c|}
\hline \multirow{3}{*}{ Compound } & \multicolumn{10}{|c|}{$\begin{array}{l}\text { Absorbance of } 1 \times 10^{-3} \mathrm{M} \text { Compound at wavelength (nm) } \\
\text { Change in Absorbance and } \lambda \text { max of Compounds at Different Concentration of DNA }\end{array}$} \\
\hline & \multirow{2}{*}{$\begin{array}{l}\lambda \max \\
(\mathrm{nm})\end{array}$} & \multirow[t]{2}{*}{ Abs. } & \multicolumn{2}{|c|}{$\begin{array}{l}1.64 \times 10^{-5} \mathrm{M}(50 \mu \mathrm{L} \text { of I } \\
\left.\times 10^{-3} \mathrm{M} \text { DNA }\right) \\
\end{array}$} & \multicolumn{2}{|c|}{$\begin{array}{l}3.23 \times 10^{-5} \mathrm{M}(100 \mu \mathrm{L} \text { of } \\
\mathrm{I} \times 10^{-3} \mathrm{M} \text { DNA) }\end{array}$} & \multicolumn{2}{|c|}{ I $\times 10^{-3} M$ DNA) } & \multicolumn{2}{|c|}{ ( $\times 10^{-3} \mathrm{M}$ DNA) } \\
\hline & & & $\lambda_{\max }(\mathrm{nm})$ & Abs. & $\lambda_{\max }(\mathbf{n m})$ & Abs. & $\lambda_{\max }(\mathrm{nm})$ & Abs. & $\lambda_{\max }(\mathrm{nm})$ & Abs. \\
\hline \multirow{2}{*}{$\mathrm{HL}$} & 252 & 0.54003 & 252 & 0.50213 & 252 & 0.47504 & 252 & 0.45226 & 252 & 0.40847 \\
\hline & 312 & 0.11095 & 312 & 0.10956 & 312 & 0.10752 & 312 & 0.10923 & 311 & 0.10562 \\
\hline$\left[\mathrm{FeL}\left(\mathrm{H}_{2} \mathrm{O}\right)_{4}\right] \mathrm{Cl}_{2}$ & 252 & 0.73363 & 252 & 0.68518 & 252 & 0.63952 & 252 & 0.60076 & 252 & 0.57015 \\
\hline$\left[\right.$ FeLL' $\left.\left(\mathrm{H}_{2} \mathrm{O}\right)_{2}\right] \mathrm{Cl}$ & 252 & $0.745 \mid 2$ & 252 & 0.69617 & 252 & 0.64046 & 252 & 0.59320 & 252 & 0.54443 \\
\hline$\left[\mathrm{CuL}\left(\mathrm{H}_{2} \mathrm{O}\right)_{2}\right] \mathrm{Cl}$ & 252 & 0.80019 & 252 & 0.750 & 252 & 0.70334 & 252 & 0.65667 & 252 & 0.61140 \\
\hline [CuLL'] & 252 & 0.64684 & 252 & $0.5947 \mid$ & 252 & 0.55216 & 252 & 0.50975 & 252 & 0.46319 \\
\hline$\left[\mathrm{RuL}\left(\mathrm{H}_{2} \mathrm{O}\right)_{4}\right] \mathrm{Cl}_{2}$ & 252 & 0.57775 & 352 & 0.52640 & 252 & $0.48 I 25$ & 252 & 0.42849 & 252 & 0.39007 \\
\hline$\left[\operatorname{RuLL}^{\prime}\left(\mathrm{H}_{2} \mathrm{O}\right)_{2}\right] \mathrm{Cl}$ & 252 & 0.94813 & 252 & 0.88942 & 252 & 0.84698 & 251 & 0.79555 & 252 & 0.74472 \\
\hline
\end{tabular}

L: 3-[(4-Bromophenylamido)]propenoic acid.

L': Diethyldithiocarbamate.

Citation: Ramzan S, Saleem S, Ali S, et al. Synthesis, characterization and thermal studies of transition metal complexes with O-, S-donor ligands.J Anal Pharm Res. 2017;5(6):I-6. DOI: 10.15406/japlr.2017.05.00।60 
Table 9 The Association Constants and Gibbs Free Energies of Transition Metal Derivatives of 3-[(4-Bromophenylamido)]propenoic acid and Diethyldithiocarbamate

\begin{tabular}{|c|c|c|}
\hline Compound & $K / M^{-1}$ & $-\Delta \mathbf{G} / \mathbf{K J ~ \mathbf { ~ m o l } ^ { - 1 }}$ \\
\hline $\mathrm{HL}$ & $127.4 \times 10^{3}$ & 29.12 \\
\hline$\left[\mathrm{FeL}\left(\mathrm{H}_{2} \mathrm{O}\right)_{4}\right] \mathrm{Cl}_{2}$ & $26.31 \times 10^{3}$ & 25.22 \\
\hline$\left[\mathrm{FeLL}\left(\mathrm{H}_{2} \mathrm{O}\right)_{2}\right] \mathrm{Cl}$ & $-18.22 \times 10^{3}$ & - \\
\hline$\left[\mathrm{CuL}\left(\mathrm{H}_{2} \mathrm{O}\right)_{2}\right] \mathrm{Cl}$ & $3.62 \times 10^{3}$ & 20.30 \\
\hline [CuLL'] & $29.33 \times 10^{3}$ & 25.49 \\
\hline$\left[\operatorname{RuL}\left(\mathrm{H}_{2} \mathrm{O}\right)_{4}\right] \mathrm{Cl}_{2}$ & $8.93 \times 10^{3}$ & 22.54 \\
\hline$\left[\operatorname{RuLL}^{\prime}\left(\mathrm{H}_{2} \mathrm{O}\right)_{2}\right] \mathrm{Cl}$ & $25.87 \times 10^{3}$ & 25.18 \\
\hline
\end{tabular}

L: 3-[(4-Bromophenylamido)]propenoic acid.

L': Diethyldithiocarbamate.

Table 10 Bond angles $\left({ }^{\circ}\right)$ of $\left[\mathrm{CrLL}^{\prime}\left(\mathrm{H}_{2} \mathrm{O}\right) \mathrm{Cl}\right]$

\begin{tabular}{|c|c|c|c|c|c|c|c|}
\hline Atom I & Atom 2 & Atom3 & Bond Angle (deg) & Atom I & Atom2 & Atom 3 & Bond Angle (deg) \\
\hline $\mathrm{O} 2$ & $\mathrm{Crl}$ & $\mathrm{O} 23$ & 63.2 & O7 & $\mathrm{C} 6$ & C5 & 123.5 \\
\hline $\mathrm{O} 2$ & $\mathrm{Crl}$ & $\mathrm{S} 24$ & 104.8 & N8 & $\mathrm{C} 6$ & C5 & 112.8 \\
\hline $\mathrm{O} 2$ & $\mathrm{Crl}$ & $\mathrm{S} 26$ & 161.9 & C6 & N8 & C9 & 126.0 \\
\hline $\mathrm{O} 2$ & $\mathrm{Crl}$ & $\mathrm{Cl} 42$ & 86.7 & $\mathrm{ClO}$ & C9 & N 8 & 117.3 \\
\hline $\mathrm{O} 2$ & $\mathrm{Crl}$ & O43 & 90.5 & $\mathrm{ClO}$ & C9 & $\mathrm{Cl} 4$ & 120.3 \\
\hline $\mathrm{O} 23$ & $\mathrm{Crl}$ & S24 & 161.7 & N8 & C9 & $\mathrm{Cl} 4$ & 122.4 \\
\hline $\mathrm{O} 23$ & $\mathrm{Crl}$ & $\mathrm{S} 26$ & 105.4 & C9 & $\mathrm{ClO}$ & $\mathrm{CII}$ & 119.7 \\
\hline $\mathrm{O} 23$ & $\mathrm{Crl}$ & $\mathrm{Cl} 42$ & 86.4 & $\mathrm{Cl} 2$ & $\mathrm{CII}$ & $\mathrm{CIO}$ & 119.5 \\
\hline $\mathrm{O} 23$ & $\mathrm{CrI}$ & O43 & 90.7 & $\mathrm{Cl} 3$ & $\mathrm{Cl} 2$ & $\mathrm{CII}$ & 121.3 \\
\hline S24 & $\mathrm{Crl}$ & S26 & 82.3 & $\mathrm{Cl} 3$ & $\mathrm{Cl} 2$ & $\mathrm{BrI7}$ & 119.4 \\
\hline S24 & $\mathrm{Crl}$ & $\mathrm{Cl} 42$ & 107.5 & $\mathrm{CII}$ & $\mathrm{Cl} 2$ & $\mathrm{BrI7}$ & 119.3 \\
\hline S24 & $\mathrm{CrI}$ & O43 & 75.0 & $\mathrm{Cl} 4$ & $\mathrm{Cl} 3$ & $\mathrm{Cl} 2$ & 119.6 \\
\hline S26 & $\mathrm{Crl}$ & $\mathrm{Cl} 42$ & 107.4 & C9 & $\mathrm{Cl} 4$ & $\mathrm{Cl} 3$ & 119.6 \\
\hline S26 & $\mathrm{Crl}$ & O43 & 75.0 & C3 & O23 & $\mathrm{Crl}$ & 88.4 \\
\hline $\mathrm{Cl} 42$ & $\mathrm{Crl}$ & O43 & 176.6 & $\mathrm{Crl}$ & S24 & $C 25$ & 82.0 \\
\hline $\mathrm{C} 3$ & $\mathrm{O} 2$ & $\mathrm{Crl}$ & 88.5 & S24 & $\mathrm{C} 25$ & S26 & 113.7 \\
\hline C4 & C3 & O23 & 121.8 & S24 & C25 & N27 & 123.1 \\
\hline C4 & C3 & $\mathrm{O} 2$ & 120.0 & S26 & $\mathrm{C} 25$ & N27 & 123.2 \\
\hline $\mathrm{O} 23$ & C3 & $\mathrm{O} 2$ & 118.2 & $\mathrm{Crl}$ & S26 & $\mathrm{C} 25$ & 82.0 \\
\hline C5 & C4 & C3 & 121.5 & C25 & N27 & C28 & 121.7 \\
\hline C6 & C5 & $\mathrm{C} 4$ & 123.2 & C25 & N27 & C35 & 121.7 \\
\hline O7 & C6 & N8 & 123.7 & C28 & N27 & C35 & 116.0 \\
\hline N27 & C28 & C29 & 111.7 & N27 & C35 & $\mathrm{C} 36$ & 111.6 \\
\hline
\end{tabular}

Table I I Bond lengths $(\AA)$ of $\left[\mathrm{CrLL}^{\prime}\left(\mathrm{H}_{2} \mathrm{O}\right) \mathrm{Cl}\right]$

\begin{tabular}{|c|c|c|c|c|c|}
\hline Atom I & Atom 2 & Bond Length (A) & Atom I & Atom 2 & Bond Length $(\AA)$ \\
\hline O7 & $\mathrm{C} 6$ & 1.21 & $\mathrm{BrI7}$ & $\mathrm{Cl} 2$ & 1.91 \\
\hline C6 & N8 & $1.4 \mathrm{I}$ & $\mathrm{Crl}$ & $\mathrm{O} 2$ & 2.10 \\
\hline $\mathrm{C} 6$ & C5 & 1.50 & $\mathrm{Crl}$ & $\mathrm{O} 23$ & 2.11 \\
\hline $\mathrm{C} 5$ & $\mathrm{C} 4$ & 1.33 & $\mathrm{~S} 24$ & $\mathrm{Crl}$ & 2.21 \\
\hline C4 & $\mathrm{C} 3$ & 1.49 & $\mathrm{Crl}$ & $\mathrm{S} 26$ & 2.21 \\
\hline $\mathrm{O} 23$ & C3 & 1.28 & $\mathrm{Cl} 42$ & $\mathrm{CrI}$ & 2.09 \\
\hline $\mathrm{C} 3$ & $\mathrm{O} 2$ & 1.28 & $\mathrm{CrI}$ & O43 & 2.21 \\
\hline $\mathrm{CIO}$ & C9 & $\mathrm{I} .4 \mathrm{I}$ & $\mathrm{C} 25$ & S24 & 1.73 \\
\hline $\mathrm{C9}$ & N8 & 1.43 & $\mathrm{C} 25$ & $\mathrm{~S} 26$ & 1.73 \\
\hline $\mathrm{C9}$ & $\mathrm{Cl} 4$ & $1.4 \mathrm{I}$ & N27 & $\mathrm{C} 25$ & 1.33 \\
\hline $\mathrm{Cl} 4$ & $\mathrm{Cl} 3$ & 1.40 & N27 & $\mathrm{C} 28$ & 1.50 \\
\hline $\mathrm{Cl} 3$ & $\mathrm{Cl} 2$ & 1.40 & N27 & C35 & 1.50 \\
\hline $\mathrm{Cl} 2$ & $\mathrm{CII}$ & $\mathrm{I} .40$ & C35 & C36 & 1.52 \\
\hline $\mathrm{Cll}$ & $\mathrm{ClO}$ & 1.39 & C28 & C29 & 1.52 \\
\hline
\end{tabular}

Table 12 Bond angles $\left({ }^{\circ}\right)$ of $\left[\mathrm{CoL}\left(\mathrm{H}_{2} \mathrm{O}\right)_{2} \mathrm{Cl}_{2}\right]$

\begin{tabular}{|c|c|c|c|c|c|c|c|}
\hline Atom I & Atom 2 & Atom 3 & Bond Angle $\left({ }^{\circ}\right)$ & Atom I & Atom2 & Atom 3 & Bond Angle $\left({ }^{\circ}\right)$ \\
\hline $\mathrm{O} 2$ & Col & $\mathrm{O} 23$ & 62.2 & N8 & $\mathrm{C} 6$ & C5 & 112.6 \\
\hline $\mathrm{O} 2$ & Col & $\mathrm{Cl} 24$ & 111.9 & $\mathrm{C} 6$ & N8 & C9 & 126.1 \\
\hline $\mathrm{O} 2$ & Col & $\mathrm{O} 25$ & 166.4 & $\mathrm{ClO}$ & $\mathrm{C9}$ & N8 & 117.4 \\
\hline $\mathrm{O} 2$ & Col & $\mathrm{Cl} 28$ & 111.6 & $\mathrm{ClO}$ & C9 & $\mathrm{Cl} 4$ & 120.1 \\
\hline $\mathrm{O} 2$ & Col & 029 & 66.5 & N8 & C9 & $\mathrm{Cl} 4$ & 122.5 \\
\hline $\mathrm{O} 23$ & Col & $\mathrm{Cl} 24$ & 137.2 & C9 & $\mathrm{ClO}$ & $\mathrm{Cll}$ & 119.8 \\
\hline $\mathrm{O} 23$ & Col & $\mathrm{O} 25$ & 105.2 & $\mathrm{Cl} 2$ & $\mathrm{CII}$ & $\mathrm{ClO}$ & 119.4 \\
\hline $\mathrm{O} 23$ & Col & $\mathrm{Cl} 28$ & 113.6 & $\mathrm{Cl} 3$ & $\mathrm{Cl} 2$ & $\mathrm{Cll}$ & 121.4 \\
\hline
\end{tabular}


Table Continued..

\begin{tabular}{|c|c|c|c|c|c|c|c|}
\hline Atom I & Atom 2 & Atom3 & Bond Angle $\left({ }^{\circ}\right)$ & Atom I & Atom 2 & Atom 3 & Bond Angle $\left({ }^{\circ}\right)$ \\
\hline $\bar{O} 23$ & Col & O29 & 66.0 & $\mathrm{Cl} 3$ & $\mathrm{Cl} 2$ & $\mathrm{BrI7}$ & 119.4 \\
\hline $\mathrm{Cl} 24$ & Col & $\mathrm{O} 25$ & 73.2 & $\mathrm{CII}$ & $\mathrm{Cl} 2$ & $\mathrm{BrI7}$ & 119.2 \\
\hline $\mathrm{Cl} 24$ & Col & $\mathrm{Cl} 28$ & 107.8 & $\mathrm{Cl} 4$ & $\mathrm{Cl} 3$ & $\mathrm{Cl} 2$ & 119.6 \\
\hline $\mathrm{Cl} 24$ & Col & O29 & 73.0 & C9 & $\mathrm{Cl} 4$ & $\mathrm{Cl} 3$ & 119.8 \\
\hline $\mathrm{O} 25$ & Col & $\mathrm{Cl} 28$ & 77.4 & C3 & $\mathrm{O} 23$ & Col & 90.6 \\
\hline $\mathrm{O} 25$ & Col & O29 & 104.6 & C5 & $\mathrm{C} 4$ & C3 & 121.4 \\
\hline $\mathrm{Cl} 28$ & Col & O29 & |78.| & $\mathrm{C} 6$ & C5 & $\mathrm{C} 4$ & 123.4 \\
\hline $\mathrm{C} 3$ & $\mathrm{O} 2$ & Col & 87.9 & O7 & $\mathrm{C} 6$ & N8 & 123.1 \\
\hline $\mathrm{C} 4$ & $\mathrm{C} 3$ & $\mathrm{O} 23$ & 117.6 & O7 & C6 & C5 & 124.3 \\
\hline $\mathrm{C} 4$ & $\mathrm{C} 3$ & $\mathrm{O} 2$ & 124.0 & $\mathrm{O} 23$ & $\mathrm{C} 3$ & $\mathrm{O} 2$ & 118.4 \\
\hline
\end{tabular}

Table 13 Bond lengths $(\AA)$ of $\left[\mathrm{CoL}\left(\mathrm{H}_{2} \mathrm{O}\right)_{2} \mathrm{Cl}_{2}\right]$

\begin{tabular}{|c|c|c|c|c|c|}
\hline Atom I & Atom 2 & Bond Length (A) & Atom I & Atom 2 & Bond Length (A) \\
\hline 07 & $\mathrm{C} 6$ & $\mathrm{I} .2 \mathrm{I}$ & $\mathrm{Cl} 2$ & $\mathrm{CII}$ & 1.40 \\
\hline C6 & N8 & $\mathrm{I} .42$ & $\mathrm{CII}$ & $\mathrm{ClO}$ & 1.39 \\
\hline C6 & C5 & 1.50 & $\mathrm{BrI7}$ & $\mathrm{Cl} 2$ & 1.91 \\
\hline C5 & $\mathrm{C} 4$ & 1.33 & Col & $\mathrm{O} 2$ & 2.17 \\
\hline C4 & $\mathrm{C} 3$ & 1.50 & Col & $\mathrm{O} 23$ & 2.09 \\
\hline $\mathrm{O} 23$ & $\mathrm{C} 3$ & 1.30 & $\mathrm{Cl} 24$ & Col & 2.43 \\
\hline $\mathrm{C} 3$ & $\mathrm{O} 2$ & 1.26 & Col & $\mathrm{O} 25$ & 2.14 \\
\hline $\mathrm{ClO}$ & C9 & $\mathrm{I} .4 \mathrm{I}$ & $\mathrm{Cl} 28$ & Col & 2.27 \\
\hline C9 & N8 & $\mathrm{I} .43$ & Col & O29 & 2.15 \\
\hline C9 & $\mathrm{Cl} 4$ & $\mathrm{I} .4 \mathrm{I}$ & $\mathrm{Cl} 3$ & $\mathrm{Cl} 2$ & 1.40 \\
\hline $\mathrm{Cl} 4$ & $\mathrm{Cl} 3$ & 1.39 & & & \\
\hline
\end{tabular}

Table 14 Bond angles $\left({ }^{\circ}\right)$ of $\left[\mathrm{CoLL}\left(\mathrm{H}_{2} \mathrm{O}\right) \mathrm{Cl}\right]$

\begin{tabular}{|c|c|c|c|c|c|c|c|}
\hline Atom I & Atom 2 & Atom3 & Bond Angle $\left({ }^{\circ}\right)$ & Atom I & Atom 2 & Atom 3 & Bond Angle $\left({ }^{\circ}\right)$ \\
\hline $\mathrm{O} 2$ & Col & $\mathrm{O} 23$ & 64.3 & N8 & $\mathrm{C} 6$ & C5 & $\mathrm{I} 12.5$ \\
\hline $\mathrm{O} 2$ & Col & $\mathrm{S} 24$ & 108.4 & $\mathrm{C} 6$ & N8 & C9 & 126.3 \\
\hline $\mathrm{O} 2$ & Col & S26 & 171.7 & $\mathrm{ClO}$ & C9 & N8 & 116.8 \\
\hline $\mathrm{O} 2$ & Col & $\mathrm{Cl} 42$ & 76.9 & $\mathrm{ClO}$ & C9 & $\mathrm{Cl} 4$ & 119.0 \\
\hline $\mathrm{O} 2$ & Col & 043 & 103.7 & N8 & C9 & $\mathrm{Cl} 4$ & 124.2 \\
\hline $\mathrm{O} 23$ & Col & $\mathrm{S} 24$ & 171.2 & C9 & $\mathrm{ClO}$ & $\mathrm{CII}$ & 119.6 \\
\hline $\mathrm{O} 23$ & Col & $\mathrm{S} 26$ & 109.0 & $\mathrm{Cl} 2$ & $\mathrm{Cll}$ & $\mathrm{ClO}$ & 119.7 \\
\hline $\mathrm{O} 23$ & Col & $\mathrm{Cl} 42$ & 76.6 & $\mathrm{Cl} 3$ & $\mathrm{Cl} 2$ & $\mathrm{CII}$ & 121.7 \\
\hline $\mathrm{O} 23$ & Col & O43 & 103.9 & $\mathrm{Cl} 3$ & $\mathrm{Cl} 2$ & $\mathrm{BrI7}$ & 118.2 \\
\hline S24 & Col & $\mathrm{S} 26$ & 77.7 & $\mathrm{Cll}$ & $\mathrm{Cl} 2$ & $\mathrm{BrI7}$ & 120.1 \\
\hline S24 & Col & $\mathrm{Cl} 42$ & 107.3 & $\mathrm{Cl} 4$ & $\mathrm{Cl} 3$ & $\mathrm{Cl} 2$ & 119.4 \\
\hline S24 & Col & O43 & 72.4 & C9 & $\mathrm{Cl} 4$ & $\mathrm{Cl} 3$ & 120.6 \\
\hline S26 & Col & $\mathrm{Cl} 42$ & 107.0 & C3 & O23 & Col & 87.9 \\
\hline S26 & Col & 043 & 72.5 & Col & $\mathrm{S} 24$ & $\mathrm{C} 25$ & 87.9 \\
\hline $\mathrm{Cl} 42$ & Col & 043 & 179.3 & $\mathrm{~S} 24$ & $\mathrm{C} 25$ & S26 & 106.2 \\
\hline C3 & $\mathrm{O} 2$ & Col & 88.3 & S24 & $\mathrm{C} 25$ & N27 & 126.9 \\
\hline C4 & $\mathrm{C} 3$ & O23 & 122.4 & S26 & C25 & N27 & 126.9 \\
\hline C4 & C3 & $\mathrm{O} 2$ & 119.4 & Col & $\mathrm{S} 26$ & $\mathrm{C} 25$ & 87.9 \\
\hline $\mathrm{O} 23$ & $\mathrm{C} 3$ & $\mathrm{O} 2$ & 118.2 & C25 & N27 & $C 28$ & 121.3 \\
\hline $\mathrm{C} 5$ & C4 & C3 & 121.8 & C25 & N27 & C35 & 121.3 \\
\hline C6 & C5 & C4 & 123.5 & C28 & N27 & C35 & 116.8 \\
\hline O7 & $\mathrm{C} 6$ & N8 & 122.6 & N27 & C28 & C29 & 111.9 \\
\hline O7 & C6 & $\mathrm{C} 5$ & 125.0 & N27 & C35 & C36 & 111.9 \\
\hline
\end{tabular}

Table 15 Bond lengths $(\AA)$ of $\left[\mathrm{CoLL}^{\prime}\left(\mathrm{H}_{2} \mathrm{O}\right) \mathrm{Cl}\right]$

\begin{tabular}{|c|c|c|c|c|c|}
\hline Atom I & Atom 2 & Bond Length (A) & Atom I & Atom 2 & Bond Length (A) \\
\hline 07 & $\mathrm{C} 6$ & 1.21 & $\mathrm{BrI7}$ & $\mathrm{Cl} 2$ & 1.90 \\
\hline C6 & N8 & $\mathrm{I} .43$ & Col & $\mathrm{O} 2$ & 2.06 \\
\hline $\mathrm{C} 6$ & C5 & 1.49 & Col & $\mathrm{O} 23$ & 2.07 \\
\hline C5 & $\mathrm{C} 4$ & 1.33 & S24 & Col & 2.19 \\
\hline C4 & C3 & 1.50 & Col & $S 26$ & 2.19 \\
\hline $\mathrm{O} 23$ & $\mathrm{C} 3$ & 1.28 & $\mathrm{Cl} 42$ & Col & 2.47 \\
\hline C3 & $\mathrm{O} 2$ & 1.28 & Col & O43 & 2.31 \\
\hline $\mathrm{ClO}$ & C9 & 1.49 & $\mathrm{C} 25$ & S24 & 1.72 \\
\hline C9 & N8 & 1.39 & $\mathrm{C} 25$ & $\mathrm{~S} 26$ & 1.72 \\
\hline $\mathrm{C9}$ & $\mathrm{Cl} 4$ & $\mathrm{I} .44$ & N27 & $\mathrm{C} 25$ & 1.33 \\
\hline $\mathrm{Cl} 4$ & $\mathrm{Cl} 3$ & 1.35 & N27 & $\mathrm{C} 28$ & 1.50 \\
\hline $\mathrm{Cl} 3$ & $\mathrm{Cl} 2$ & 1.46 & N27 & C35 & 1.50 \\
\hline $\mathrm{Cl} 2$ & $\mathrm{CII}$ & $\mathrm{I} .4 \mathrm{I}$ & C35 & C36 & 1.52 \\
\hline $\mathrm{Cll}$ & $\mathrm{ClO}$ & 1.37 & C28 & $\mathrm{C} 29$ & 1.52 \\
\hline
\end{tabular}




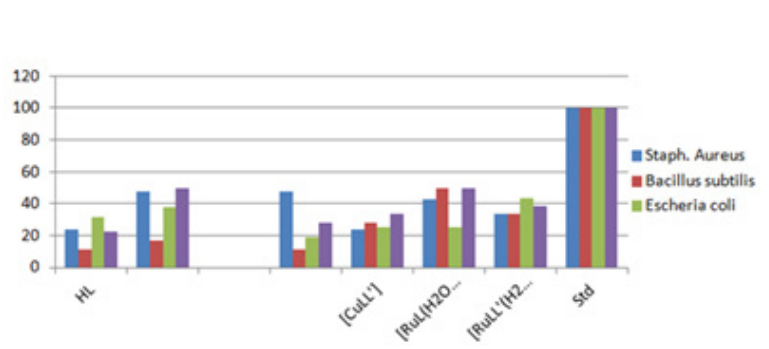

Figure 5 Graphical representation of antibacterial activity of selected compounds against four types of bacteria.

$\mathrm{H}_{2} \mathrm{O}_{2}$ induced DNA damage assay: Antioxidant and pro-oxidant effects of all synthesized compounds were investigated in vitro by using a DNA protection assay. With the attack of hydroxyl radicals generated in the Fenton reaction, if scission occurs on one strand (single stranded nicking) of plasmid DNA, the supercoiled (SC) form will be relaxed to generate a slower-moving open circular (OC) form. If both strands of the plasmid DNA are cleaved (double stranded nicking), a linear form that migrates between the open circular form and the supercoiled form will be generated. Thus, the ability of the test compounds to unwind or condense a supercoiling substrate such as a plasmid DNA was examined. In the present study, it was found that compounds $\quad\left[\mathrm{FeL}\left(\mathrm{H}_{2} \mathrm{O}\right)_{4}\right] \mathrm{Cl}_{2}, \quad\left[\mathrm{CuL}\left(\mathrm{H}_{2} \mathrm{O}\right)_{2}\right]$ Cland[CuLL']enhanced DNA damage and completely converted the supercoiled DNA into the linear and open circular forms. ${ }^{29}$ Most compounds enhanced the DNA damage at $1000 \mathrm{ppm}$ concentrations as compared to lower concentrations $(10,100 \mathrm{ppm})$ (Table 7). 3-[(4-Bromophenylamido)] propenoic acid protected the plasmid DNA at all concentrations (10, $100,1000 \mathrm{ppm})$. This conclusion was deduced by the disappearance or reduction in the initial quantity of DNA (Figure 6-8).
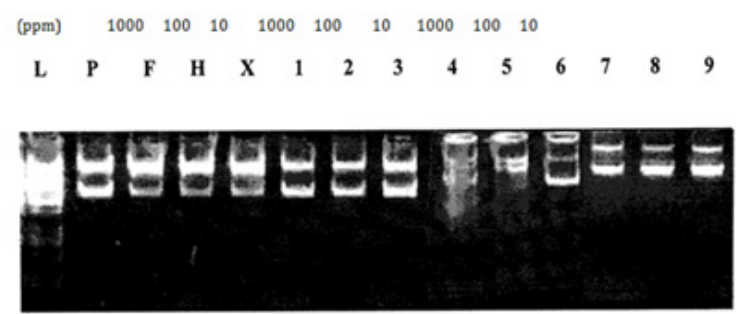

Figure 6 Effect of $\mathrm{HL}(\mathrm{I}-3)$; compound $\left[\mathrm{FeL}\left(\mathrm{H}_{2} \mathrm{O}\right)_{4}\right] \mathrm{Cl}_{2}(4-6)$ and compound [FeLL' $\left.\left(\mathrm{H}_{2} \mathrm{O}\right)_{2}\right] \mathrm{Cl}$ (7-9) on pBR322 plasmid DNA.
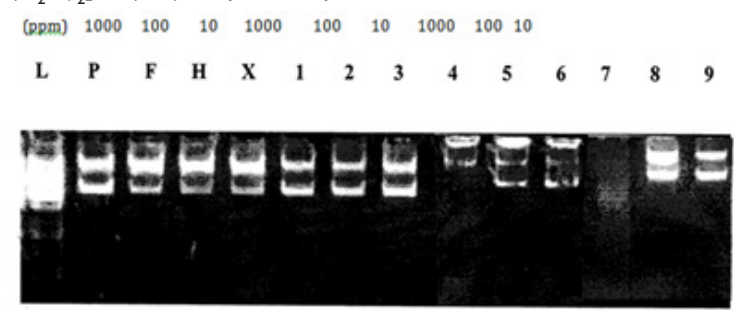

Figure 7 Effect of $\mathrm{HL}(\mathrm{I}-3)$, compound $\left[\mathrm{CuL}\left(\mathrm{H}_{2} \mathrm{O}\right)_{2}\right] \mathrm{Cl}(4-6)$ and compound [CuLL'] (7-9) on pBR322 plasmid DNA.

$\begin{array}{llllllllll}\text { (ppm) } & 1000 & 100 & 10 & 1000 & 100 & 10 & 1000 & 100 & 10\end{array}$
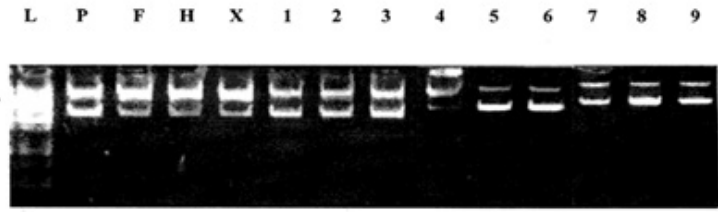

Figure 8 Effect of $\mathrm{HL}(\mathrm{I}-3)$, compound $\left[\mathrm{RuL}\left(\mathrm{H}_{2} \mathrm{O}\right)_{4}\right] \mathrm{Cl}_{2}(4-6)$ and compound $\left[\mathrm{RuL}\left(\mathrm{H}_{2} \mathrm{O}\right)_{4}\right] \mathrm{Cl}_{2}(7-9)$ on pBR322 plasmid DNA.
L: IKB Ladder

$\mathrm{P}: \mathrm{pBr} 322$ Plasmid

$\mathrm{F}: \mathrm{pBr} 322$ Plasmid treated with $\mathrm{FeSO}_{4}$

$\mathrm{H}: \mathrm{pBr} 322$ Plasmid treated with $\mathrm{H}_{2} \mathrm{O}_{2}$

$\mathrm{X}: \mathrm{pBr} 322$ Plasmid treated with $\mathrm{FeSO}_{4} \& \mathrm{H}_{2} \mathrm{O}$

DNA interaction by UV/visible spectroscopy: The interactions of selected transition metal derivatives of 3-[(4-bromophenylamido)] propenoic acid and diethyldithiocarbamate were also examined by UV-Vis. spectroscopy in order to study the mode of binding with DNA. The hypochromic effect is observed in all cases, which is caused by the overlapping of electronic states of the chromophores of the complexes with DNA bases (Table 8). The binding constants were calculated according to the Benesi-Hildebrand equation. ${ }^{30}$ The slope to intercept ratio of the plot between $A_{0} /\left(A-A_{0}\right)$ verses $1 /[\mathrm{DNA}]$ gives the binding constants. The plot between $A_{0} /\left(A-A_{0}\right)$ verses $1 /[\mathrm{DNA}]$ for the complex $\left[\mathrm{CuL}\left(\mathrm{H}_{2} \mathrm{O}\right)_{2}\right] \mathrm{Cl}$ is shown in Figure 9 whereas the values of binding constants for the rest of the complexes is given in Table 9.

\section{Constant of $\left[\mathrm{CuL}\left(\mathrm{H}_{2} \mathrm{O}\right)_{2}\right] \mathrm{Cl}$ - DNA adduct}

Semi empirical study: Semi empirical studies of selected compounds were conducted in which the molecules in the gas phase were modeled by a MOPAC $2007^{31}$ program using PM6 method. ${ }^{32}$ Selected parts of the complexes not containing the metal ion were pre optimized using molecular mechanics methods. Several cycles of energy minimizations had to be carried out for each of the molecules. The molecular mechanics correction was applied to the - $\mathrm{Co}-\mathrm{NH}-$ barrier. The geometry was optimized using Eigen Vectors. The Root Mean Square Gradients for molecules were all less than one. Self Consistent Field was achieved in each case (Figure 10).

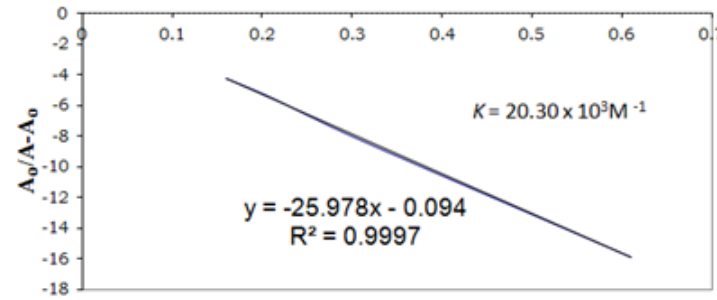

$1 /[\mathrm{DNA}] /(\mu \mathrm{M})^{-1}$

Figure 9 Plot of $A_{0}\left(A-A_{0}\right)$ vs. $1 /[D N A]$ for the determination of binding constant of $\left[\mathrm{CuL}\left(\mathrm{H}_{2} \mathrm{O}\right)_{2}\right] \mathrm{Cl}$ - DNA adduct.

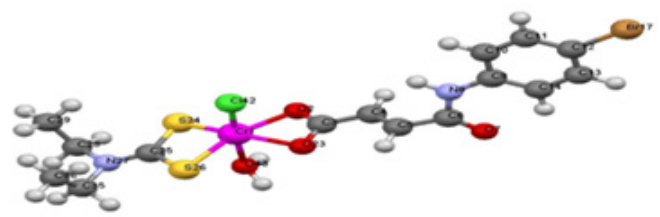

Figure 10 Optimized structure of [CrLL'( $\left.\left.\mathrm{H}_{2} \mathrm{O}\right) \mathrm{Cl}\right]$.

Semi Empirical Study of $\left[\operatorname{CrLL}^{\prime}\left(\mathrm{H}_{2} \mathrm{O}\right) \mathbf{C l}\right]$ : The diethyldithiocarbamate and 3-[(4-bromophenylamido)]propionate ligands bind through the sulphur and oxygen atoms in the equatorial plane. The S-Cr-S angle is $82.3 \mathrm{deg}$ while the $\mathrm{O}-\mathrm{Cr}-\mathrm{O}$ angle is 63.2 deg. The computed $\mathrm{Cr}-\mathrm{S}$ distance is $2.21 \mathrm{~A}^{033}$ and $\mathrm{Cr}-\mathrm{O}$ (lig) distance is $2.10 \mathrm{~A}^{\circ} .{ }^{34}$ The apical position is occupied by a chloride ion and a water molecule occupies the other axial position and completes the coordination sphere of the chromium ion. The computed $\mathrm{Cr}-\mathrm{Cl}$ distance is $2.09^{35}$ while the $\mathrm{Cr}-\mathrm{O}\left(\mathrm{H}_{2} \mathrm{O}\right)$ is $2.11 \mathrm{~A}^{\circ}{ }^{36}$ In the dithocarbamate 
ligand the $\mathrm{S}-\mathrm{C}$ bond length is $1.73 \mathrm{~A}^{\circ} .{ }^{37}$ The $\mathrm{Cl}-\mathrm{Cr}-\mathrm{O}\left(\mathrm{H}_{2} \mathrm{O}\right)$ angle is $176.6 \mathrm{deg}$. The chromium ion is $0.29 \mathrm{~A}^{\circ}$ above the mean plane of the $\mathrm{S} 2 \mathrm{O} 2$ coordinating plane. The bond angles and bond lengths are given in the Tables $10 \& 11$ (Figure 11).

Semi empirical structure of $\left[\mathrm{CoL}\left(\mathrm{H}_{2} \mathrm{O}\right)_{2} \mathrm{Cl}_{2}\right]:$ The diethyldithiocarbamato ligand binds to cobalt through the carboxylate oxygen atoms in the equatorial plane. The O-Co-O angle is $62.2^{\circ}$. The computed Co-O distances are 2.08 and $2.17 \AA .{ }^{38}$ The other two equatorial sites are occupied by a water molecule and a $\mathrm{Cl}$ ion. The apical position is occupied by a chloride ion and a water molecule occupies the other axial position and completes the distorted octahedral coordination sphere of the cobalt ion. The computed $\mathrm{Co}-\mathrm{Cl}$ distance is $2.27,2.43 \AA{ }^{39}$ while the $\mathrm{Co}-\mathrm{O}\left(\mathrm{H}_{2} \mathrm{O}\right) 2.14,2.15 \AA .{ }^{38}$ The $\mathrm{Cl}-$ $\mathrm{Co}-\mathrm{O}\left(\mathrm{H}_{2} \mathrm{O}\right)$ angle is $178.1^{\circ}$. The cobalt ion is $0.5 \mathrm{~A}^{\circ}$ above the mean plane equatorial plane. The bond angles and bond lengths are given in the Tables $12 \& 13$, respectively (Figure 12).

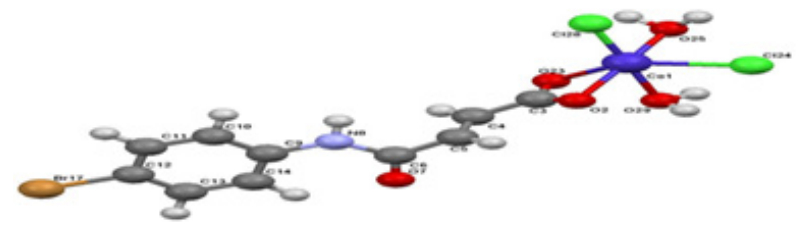

Figure I I Optimized structure of $\left[\mathrm{CoL}\left(\mathrm{H}_{2} \mathrm{O}\right)_{2} \mathrm{Cl}_{2}\right]$.

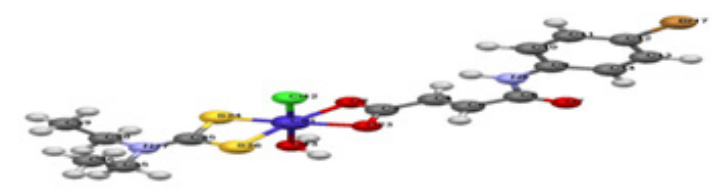

Figure 12 Optimized structure of [CoLL' $\left.\left(\mathrm{H}_{2} \mathrm{O}\right) \mathrm{Cl}\right]$.

Semi Empirical Structure of $\left[\mathrm{CoLL}^{\prime}\left(\mathrm{H}_{2} \mathrm{O}\right) \mathbf{C l}\right]$ : The diethyldithiocarbamato and 4-bromoanilinopropenoiato ligands bind through the sulphur and oxygen atoms in the equatorial plane. The $\mathrm{S}-\mathrm{Co}-\mathrm{S}$ angle is $77.7^{\circ}$, while the $\mathrm{O}-\mathrm{Co}-\mathrm{O}$ angle is $64.3^{\circ}$. The Co-S distance is $2.19 \AA^{40}$ and the Co-O (lig) distance is $2.06 \AA .^{38}$ The apical position is occupied by a chloride ion and a water molecule occupies the trans axial position and completes the coordination sphere of the chromium ion. The $\mathrm{Co}-\mathrm{Cl}$ distance is $2.47,{ }^{39}$ while the $\mathrm{Co}-\mathrm{O}\left(\mathrm{H}_{2} \mathrm{O}\right)$ $2.31 \AA .{ }^{38}$ The $\mathrm{Cl}-\mathrm{Co}-\mathrm{O}\left(\mathrm{H}_{2} \mathrm{O}\right)$ angle is $179.3^{\circ}$. The cobalt ion is $0.1 \AA$ above the mean plane of the $\mathrm{S} 2 \mathrm{O} 2$ coordinating plane. The bond angles and bond lengths are given in the Table $14 \& 15$ respectively.

Transition metal derivatives have been synthesized in quantitative yields by refluxing carboxylic acids and respective transition metal chlorides in dry ethanol. The FT-IR spectra clearly demonstrate that the $[\mathrm{O}, \mathrm{O}]$ binding sites of the ligand coordinate to the metal and behave as bidentate ligands. Moreover, the appearance of new peaks in the region of $500-400 \mathrm{~cm}^{-1}$ is due to $\mathrm{M}-\mathrm{O}$ stretching mode which confirms that the metal has coordinated to the ligand. Semi empirical studies of complexes show that the $\mathrm{Cr}$ and $\mathrm{Co}$ derivatives are six coordinated, imparting octahedral geometry which is in accordance with the structure proposed by other spectroscopic results. Decomposition reactions of the complexes occur in one, two or three steps leaving only the metal oxide as residual product at $900-1000^{\circ} \mathrm{C}$. Kinetic studies of all complexes indicate first order reactions. The activation energies for the first step is less compared with the second step. The same trend is valid for the enthalpy of the reactions. Biological activity data show that all complexes are biologically active with a few exceptions. $\mathrm{Cu}$ and $\mathrm{Fe}$ complexes of 3-[(4-bromophenylamido)]propenoic acid destroy DNA a little at higher concentrations whereas the Ru complex protects it. It can be assumed that after testing against various other cancer models and at different doses these compounds may prove to be safer anticancer drugs for tomorrow. 4-Bromoanilinopropenoic acid showed more binding strength with DNA as compared to its metal derivatives. The increasing order is $\left[\mathrm{CuL}\left(\mathrm{H}_{2} \mathrm{O}\right)_{2}\right] \mathrm{Cl}<\left[\mathrm{RuLL}^{\prime}\left(\mathrm{H}_{2} \mathrm{O}\right)_{2}\right] \mathrm{Cl}<\left[\mathrm{FeL}\left(\mathrm{H}_{2} \mathrm{O}\right)_{4}\right]$ $\mathrm{Cl}_{2}<\left[\mathrm{CuLL}^{\prime}<3-[(4\right.$-bromophenylamido $)]$ propenoic acid.

\section{Acknowledgements}

Quaid-i-Azam University, Islamabad is highly acknowledged for financial support.

\section{Conflicts of Interset}

None.

\section{References}

1. Sanyan LA, Ankel C, Kirshnamurti C. Comparative cytotoxic and biochemical effects of ligands and metal complexes of .alpha.-Nheterocyclic carboxaldehyde thiosemicarbazones. J Med Chem. 1979;22(10):1218-1221.

2. Burger K, Illes J, Gyurcsik B. Metal ion coordination of macromolecular bioligands: formation of zinc(II)complex of hyaluronic acid. Carbohydr Res. 2001;332:197.

3. Mojumdar SC, Martika L, Valigura D. J Therm Anal Cal. 2005;81:243.

4. Czakis-Sulikowska D, Czylkowska A, Malinowska A. Thermal and Other Properties of New 4,4'-bipyridine-trichloroacetato Complexes of $\mathrm{Mn}(\mathrm{II}), \mathrm{Ni}(\mathrm{II})$ and $\mathrm{Zn}(\mathrm{II})$. Journal of Thermal Analysis and Calorimetry. 2002;67(3):667-678.

5. Jona E, Sapietová M, Pavlík V. Res J Chem Environ. 2007;11:23.

6. Saleh MT, Mojumdar SC, Amoureux ML. Res J Chem Environ. 2006;10:14.

7. Jóna E, Kubranová M, Imon P, Mroziski J. Thermochemical investigation. Journal of Thermal Analysis and Calorimetry. 1996;46(5):1325-1337.

8. Mojumdar SC, Madhurambal G, Saleh MT. A study on synthesis and thermal, spectral and biological properties of carboxylato-Mg(II) and carboxylato- $\mathrm{Cu}$ (II) complexes with bioactive ligands. Journal of Thermal Analysis and Calorimetry. 2006;81(1):205-210.

9. Mojumdar SC, Martika L, Valigura D, et al.Thermal and spectral properties of halogenosalicylato-Cu(II) complexes. Journal of Thermal Analysis and Calorimetry. 2003;74(3):905-910.

10. Ramadevi A, Srinivasan K. Agricultural Solid Waste for the Removal of Inorganics: Adsorption of Mercury (II) from Aqueous Solution by Tamarind Nt Carbon. Res J Chem Environ. 2003;12(4):407-412.

11. Shahzadi S, Shahid K, Ali S. Coordination behavior of the carboxylate group in organotin(IV) derivatives of 2-[(2',4',6'-tribromophenylamido)] benzoic acid and $3-\left[\left(2^{\prime}, 4^{\prime}, 6^{\prime}\right.\right.$-tribromophenylamido $\left.)\right]$ propenoic acid: Spectroscopic studies. Russian Journal of Coordination Chemistry. 2003;33(6):403-411.

12. Dele'pine M. Bull Soc Chim Franc.1958; 5.

13. Gray HB. Transition Metal Chemistry. 1956;1:239.

14. Glen K, Schwab R. Angew Chem. 1950;62:320.

15. Fackler JP, Holah DG. Inorg Nucl Chem Lett. 1996;2:251.

16. Alderman PRH, Owston PG, Rowe J. The crystal structure of nitroso(dimethyldithiocarbonato)cobalt $[\mathrm{Co}(\mathrm{NO})\{\mathrm{S} 2 \mathrm{CN}(\mathrm{CH} 3) 2\} 2] . J$ Chem Soc. 1962; p. 668.

17. Bellamy LJ. The Infrared Spectra of Complex molecules. John Wiley and Sons, New York, USA. 1958. 
18. Armarego WLF, Perrin DD. Purification of Laboratory Chemicals. $\left(4^{\text {th }}\right.$ edn), Pergamon, Oxford, USA. 1957.

19. Armarego WLF, Chai CLL. Purification of Laboratory Chemicals. $\left(5^{\text {th }}\right.$ edn), Butterworth-Heinemann, London, New York, USA. 2003

20. Shahid K, Ali S, Shahzadi S, et al. Organotin(IV) Complexes of Aniline Derivatives. I. Synthesis, Spectral and Antibacterial Studies of Di- and Triorganotin(IV) Derivatives of 4-Bromomaleanilic Acid. Journal Synthesis and Reactivity in Inorganic and Metal-Organic Chemistry. 2003;33(7):1221-1235.

21. Xie QL, Yang Z, Jiang L. Main Group Met Chem. 1996;19:509.

22. Sandhu GK, Kaur GJ. J Organomet Chem. 1990;338:63.

23. Honnick WD, Zukerman JJ. J Organomet Chem. 1979;178:133.

24. Davis AG, Smith PJ. Comprehensive Organometallic Chemistry. Wilkinson G, et al. (Eds.), Oxford: Pergamon Press, USA, 1982. p. 539.

25. Siddiqi KS, Khan S, Nami SA, et al. Spectrochim Acta A: Mol Biomol Spectrosc. 2007;67:99.

26. Siddiqi SK, Shahab AA, Nami L, et al. Template Synthesis of Symmetrical Transition Metal Dithiocarbamates. J Braz Chem Soc. 2006;17(1):107-112.

27. Horowitz HH, Metzger G. A New Analysis of Thermogravimetric Traces. Analytical Chemistry. 1963;35(10):1464-1468.

28. Coats AW, Redfern JP. Nature. 1964;68:201.

29. Tian B, Hua Y. Food Chem. 2005;91:413.
30. Ibrahim MS, Shehatta IS, Al-Nayeli AA. J Pharm Biomed Anal. 2002;28:217.

31. Stewart JJP. Stewart Computational Chemistry, MOPAC Version 7334 W. 2007.

32. Stewart JJP. Optimization of parameters for semiempirical methods V: Modification of NDDO approximations and application to 70 elements. Journal of Molecular Modeling. 2007;13(12):1173-1213.

33. Merlino S, Sartori F. Acta Crystallographica. 1972;B28:972.

34. Eshel M, Bino A, Felner I. Chem. 2000;39(7):1376.

35. Choi JH, Kim HS, Habibi MH. Bull Korean Chem Soc. 2008;29(7):1399.

36. Sajidu SMI, Persson I, Masamba WRL, et al. Mechanisms for biosorption of chromium (III), copper (II) and mercury (II) using water extracts of Moringa oleifera seed powder. African J Biotechnology. 2008;7(6):800-804.

37. Martin RL, Patrrick JM, Skelton BW, et al. Crystal structure of Bis[N,Ndiethyl(dithiocarbamato-S, $\left.\left.\mathrm{S}^{\prime}\right)\right][\mathrm{N}, \mathrm{N}$-diethyl-(dithioperoxycarbamato$\mathrm{O}, \mathrm{S})$ ]chromium(III). (A redetermination). Australian Journal of Chemistry. 1982;35(12):2551-2556.

38. Bullen GJ (1959) The crystal structure of cobalt(II) bisacetylacetone dehydrate. Acta Crystallographica 12(10): 703-708.

39. Lai Wb, Ming-Yan W, Ya-Qiong G. Chinese J Struc Chem. 2014;25(5):516.

40. Fun HK, Raj SSS, Zhu XH. Acta Crystallographica. 1999. p. 55. 